\title{
FRACTIONAL CHROMATIC NUMBER, MAXIMUM DEGREE AND GIRTH
}

\author{
FRANÇOIS PIROT AND JEAN-SÉBASTIEN SERENI
}

\begin{abstract}
We introduce a new method for computing bounds on the independence number and fractional chromatic number of classes of graphs with local constraints, and apply this method in various scenarios. We prove that if $G$ is a triangle-free graph with maximum degree $\Delta \geq 2$, then its fractional chromatic number is at most $1+\left(1+\frac{2}{\ln \Delta}\right) \frac{\Delta}{\ln \Delta-2 \ln \ln \Delta}$. This matches the best asymptotic upper bound known for off-diagonal Ramsey numbers, and is the first explicit bound of this nature. It is obtained as a corollary of a general upper bound on the fractional chromatic number of triangle-free graphs, which matches, for small values of the maximum degree $\Delta$, that deduced from the fractional version of Reed's bound for triangle-free graphs, and improves it when $\Delta \geq 17$, transitioning smoothly to the correct asymptotic regime. Focusing on smaller values of $\Delta$, we also demonstrate that every graph of girth at least 7 and maximum degree $\Delta$ has fractional chromatic number at most $1+\min _{k \in \mathbb{N}} \frac{2 \Delta+2^{k-3}}{k}$. In particular, the fractional chromatic number of a graph of girth 7 and maximum degree $\Delta$ is at most $\frac{2 \Delta+9}{5}$ when $\Delta \in[3,8]$, at most $\frac{\Delta+7}{3}$ when $\Delta \in[8,20]$, at most $\frac{2 \Delta+23}{7}$ when $\Delta \in[20,48]$, and at most $\frac{\Delta}{4}+5$ when $\Delta \in[48,112]$. In addition, we also obtain new lower bounds on the independence ratio of graphs of maximum degree $\Delta \in\{3,4,5\}$ and girth $g \in\{6, \ldots, 12\}$, notably $1 / 3$ when $(\Delta, g)=(4,10)$ and $2 / 7$ when $(\Delta, g)=(5,8)$.
\end{abstract}

\section{INTRODUCTION}

1.1. A motivation coming from Ramsey theory. Since the seminal result of Ramsey [20], the branch of combinatorics now called "Ramsey Theory" has known an ever increasing range of interest from the community.

Theorem 1 (Ramsey, 1930). For every integers $s, t \geq 2$, there exists a minimal integer $R(s, t)$ such that every graph on $n \geq R(s, t)$ vertices contains either a clique of size $s$, or an independent set of size $t$.

Computing the exact value of the Ramsey number $R(s, t)$ for all possible pairs $(s, t)$ is a notorious problem, and only little progress has been made since the first quantitative result due to Erdös and Szekeres [11] that $R(s, t) \leq\left(\begin{array}{c}s+t-2 \\ s-1\end{array}\right)$ for every $s, t \geq 2$. Two particular regimes of the Ramsey numbers have attracted a particular focus, namely the diagonal Ramsey numbers $R(s, s)$, and the off-diagonal Ramsey numbers $R(s, t)$ where $s$ is a fixed constant (typically $s=3$ ) and $t \rightarrow \infty$. The result of Erdős and Szekeres [11] implies that $\frac{1}{s} \ln R(s, s) \leq 4$, while Erdős [10] showed in 1947 through an analysis of random graphs drawn from $G\left(n, \frac{1}{2}\right)$ that $\frac{1}{s} \ln R(s, s) \geq \frac{1}{2}$. Reducing this gap is still an open problem from these days. Our work is mainly motivated by the off-diagonal regime. In this setting, it is relevant to introduce the maximum degree $\Delta(\cdot)$ as an additional parameter, since on one hand $\Delta(G)$ is an easy lower bound on the independence number of a triangle-free graph $G$, and on the other hand graphs with smaller maximum degree are easier to colour, and hence contain larger independent sets. The best general upper bound on $R(3, t)$ to this date is due to Shearer [21] and can be deduced from the following result.

Theorem 2 (Shearer, 1983). Every triangle-free graph $G$ on $n$ vertices and of average degree $d$ contains an independent set of size at least $\frac{d \ln d-d+1}{(d-1)^{2}} n$. In particular, this implies that $R(3, t) \lesssim \frac{t^{2}}{\ln t}$.

In this paper, we are interested in finding refinements of Theorem 2 . We will study the fractional chromatic number and Hall ratio of graphs of given girth. Before going further, we introduce the relevant notions. 
1.2. Definitions and observations. The fractional chromatic number $\chi_{f}(G)$ of a graph $G$ is a refinement of the chromatic number. It is the fractional solution to a linear program, the integer solution of which is the chromatic number. Let $G$ be a given graph; we define $\mathscr{I}_{\max }(G)$ to be the set of all maximal independent sets of $G$ and $\mathscr{I}_{\alpha}(G)$ to be the set of all maximum independent sets of $G$. Then $\chi_{f}(G)$ is the solution of the following linear program.

$$
\begin{gathered}
\min \sum_{I \in \mathscr{I}_{\max }(G)} w_{I} \\
\text { such that } \begin{cases}w_{I} \in[0,1] & \text { for each } I \in \mathscr{I}_{\max } \\
\sum_{\substack{I \in \mathscr{I}_{\max } \\
v \in I}} w_{I} \geq 1 & \text { for each } v \in V(G) .\end{cases}
\end{gathered}
$$

A fractional colouring of weight $w$ of $G$ is any instance within the domain of the above linear program such that $\sum w_{I}=w$. You can note that a $k$-colouring of $G$ is a special case of a fractional colouring of weight $k$ of $G$, where $w_{I}=1$ if $I$ is a monochromatic class of the $k$-colouring, and $w_{I}=0$ otherwise. Note also that if $W$ is a clique in $G$, then any fractional colouring of $G$ is of weight at least $|W|$, hence $\chi_{f}(G) \geq \omega(G)$. Another elementary lower bound on the fractional chromatic number comes from the independence number $\alpha(G)$. Indeed, the total weight induced by an independent set $I$ of $G$ on its vertex set is at most $w_{I} \alpha(G)$, and so the weight of a fractional colouring of $G$ is at least $|V(G)| / \alpha(G)$. These two lower bounds can be combined by the Hall ratio $\rho(G)$ of $G$, which is defined as

$$
\rho(G):=\max \left\{\frac{|V(H)|}{\alpha(H)}: H \subseteq G\right\} .
$$

Summing up the above observations, this allows us to write the following inequalities

$$
\max \left\{\omega(G), \frac{|V(G)|}{\alpha(G)}\right\} \leq \rho(G) \leq \chi_{f}(G) \leq \chi(G) \leq \Delta(G)+1
$$

where $\Delta(G)$ is the maximum degree of $G$. Equality holds between $\omega(G)$ and $\chi(G)$, and so in particular between $\omega(G)$ and $\chi_{f}(G)$, when $G$ is a perfect graph. Those are the graphs that contain no odd hole nor odd antihole, as was conjectured by Berge [2] in 1961, and proved by Chudnovsky et al. [7] in 2006. On the other side, the characterisation of the graphs $G$ for which equality holds between $\chi(G)$ and $\Delta(G)+1$ was established by Brooks [5] in 1941, and those graphs are cliques and odd cycles. Since $\chi_{f}\left(C_{2 k+1}\right)=\frac{k}{2 k+1}$, the only graphs $G$ such that $\chi_{f}(G)=\Delta(G)+1$ are cliques. Moreover, equality holds between the Hall ratio of $G$ and its fractional chromatic number for example when $G$ is vertex transitive.

1.3. Previous results on the Hall ratio. Since the Hall ratio is the hereditary version of the inverse of the independence ratio (defined as the independence number divided by the number of vertices), any result on the independence ratio in a hereditary class of graphs can be extended to the Hall ratio. The Hall ratio of a graph has often been studied in relation with the girth, which is the length of a smallest cycle in the graph. A first result in this direction is the celebrated introduction of the so-called "deletion method" in graph theory by Erdős, who used it to demonstrate the existence of graphs with arbitrarily large girth and chromatic number. The latter is actually established by proving that the Hall ratio of the graph is arbitrarily large. As a large girth is not strong enough a requirement to imply a constant upper bound on the chromatic number, a way to pursue this line of research is to express the upper bound in terms of the maximum degree $\Delta(G)$ of the graph $G$ considered. This also applies to the Hall ratio.

Letting girth $(G)$ stand for the girth of the graph $G$, that is, the length of a shortest cycle in $G$ if $G$ is not a forest and $+\infty$ otherwise, we define $\rho(d, g)$ to be the supremum of the Hall ratios 
among all graphs of maximum degree at most $d$ and girth at least $g$. We also let $\rho(d, \infty)$ be the limit as $g \rightarrow \infty$ of $\rho(d, g)$ - note that if we fix $d$ then $\rho(d, g)$ is a non-increasing function of $g$. In symbols,

$$
\begin{aligned}
\rho(d, g) & :=\sup \left\{\frac{|V(G)|}{\alpha(G)}: G \text { graph with } \Delta(G) \leq d \text { and } \operatorname{girth}(G) \geq g\right\}, \\
\rho(d, \infty) & :=\lim _{g \rightarrow \infty} \rho(d, g) .
\end{aligned}
$$

In 1979, Staton [23] established that $\rho(d, 4) \leq \frac{5 d-1}{5}$, in particular implying that $\rho(3,4) \leq \frac{14}{5}$. The two graphs depicted in Figure 1. called the graphs of Fajtlowicz and of Locke, have fourteen vertices each, girth 5 , and no independent set of order 6 . It follows that $\rho(3,4)=\frac{14}{5}=\rho(3,5)$. It is known that the graphs of Fajtlowicz and of Locke are the only two cubic triangle-free and connected graphs with Hall ratio $\frac{14}{5}$. This follows from a result of Fraughnaugh and Locke [13] for graphs with more than 14 vertices completed by an exhaustive computer check on graphs with at most 14 vertices performed by Bajnok and Brinkmann [1].
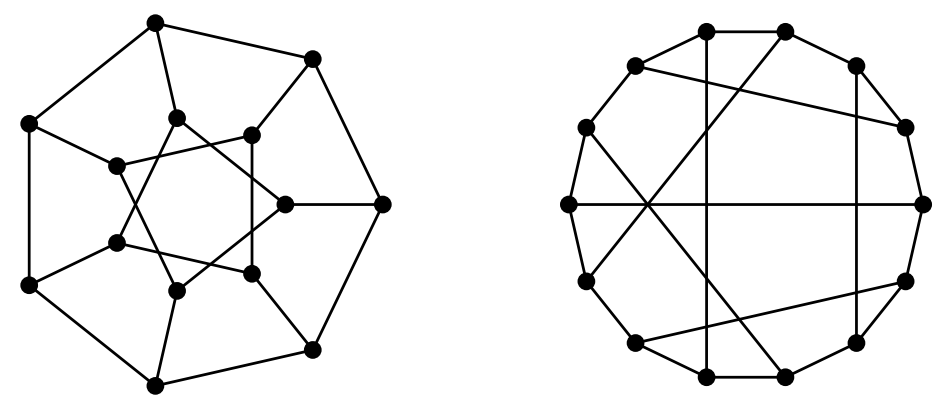

Figure 1. The two cubic triangle-free connected graphs with Hall ratio $\frac{14}{5}$.

In 1983, Jones 14 reached the next step by establishing that $\rho(4,4)=\frac{13}{4}$. Only one connected graph is known to attain this value: it has 13 vertices and is represented in Figure 2, The value of $\rho(d, 4)$ when $d \geq 5$ is still unknown; the best general upper bound is due to Shearer, and improves his first bound stated in Theorem 2. He also provides an upper bound for $\rho(d, 6)$ as a consequence of a stronger result on graphs with no cycle of length 3 or 5 .

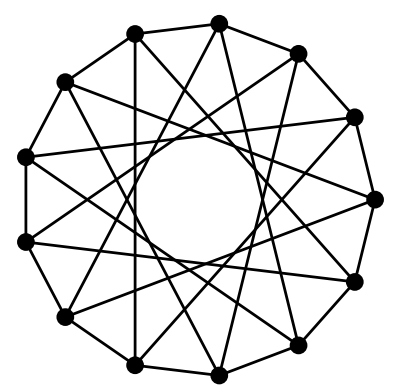

Figure 2. The only known 4-regular triangle-free connected graph of Hall ratio $\frac{13}{4}$.

Theorem 3 (Shearer [22]). Set $f(0):=1$, and $f(d):=\frac{1+\left(d^{2}-d\right) f(d-1)}{d^{2}+1}$ for every integer $d \geq 2$. If $G$ is a triangle-free graph on $n$ vertices with degree sequence $d_{1}, \ldots, d_{n}$, then $G$ contains an independent of size $\sum_{i=1}^{n} f\left(d_{i}\right)$. 
Theorem 4 (Shearer [22]). Set $f(0):=0, f(1):=\frac{4}{7}$, and $f(d):=\frac{1+\left(d^{2}-d\right) f(d-1)}{d^{2}+1}$ for every integer $d \geq 2$. If $G$ is a graph on $n$ vertices with degree sequence $d_{1}, \ldots, d_{n}$ and with no 3 -cycle and no 5 -cycle, then $G$ contains an independent of size $\sum_{i=1}^{n} f\left(d_{i}\right)-\frac{n_{11}}{7}$, where $n_{11}$ is the number of pairs of adjacent vertices of degree 1 in $G$.

Theorems 3 and 4 allow us to compute upper bounds on $\rho(d, 4)$ and on $\rho(d, 6)$ for small values of $d$, as indicated in Table 1 . When $d \geq 5$, these bounds are the best known ones.

\begin{tabular}{rrrrl}
\hline$d$ & upper bound on $\rho(d, 4)$ & upper bound on $\rho(d, 6)$ \\
\hline 2 & $\frac{5}{2}$ & $=2.5$ & $\frac{7}{3}$ & $\approx 2.33333$ \\
3 & $\frac{50}{17}$ & $\approx 2.94118$ & $\frac{14}{5}$ & $=2.8$ \\
4 & $\frac{425}{127}$ & $\approx 3.34646$ & $\frac{119}{37}$ & $\approx 3.21622$ \\
5 & $\frac{2210}{593}$ & $\approx 3.72681$ & $\frac{3094}{859}$ & $\approx 3.60186$ \\
6 & $\frac{8177}{2000}$ & $\approx 4.0885$ & $\frac{57239}{14432}$ & $\approx 3.96612$ \\
7 & $\frac{408850}{92177}$ & $\approx 4.43549$ & $\frac{408850}{94769}$ & $\approx 4.31417$ \\
8 & $\frac{13287625}{2285381}$ & $\approx 4.77049$ & $\frac{13287625}{2287957}$ & $\approx 4.64934$ \\
9 & $\frac{1089585250}{213835057}$ & $\approx 5.09545$ & $\frac{1089585250}{219006529}$ & $\approx 4.9739$ \\
10 & $\frac{11004811025}{2033474038}$ & $\approx 5.41183$ & $\frac{11004811025}{2080503286}$ & $\approx 5.28949$ \\
\hline
\end{tabular}

TABLE 1. Upper bounds on $\rho(d, 4)$ and $\rho(d, 6)$ for $d \leq 10$ derived from Theorems 3 and 4

We are not aware of any non trivial lower bounds on $\rho(5,4)$ and $\rho(6,4)$. Figure 3 show graphs illustrating that $\rho(5,4) \geq \frac{10}{3} \approx 3.33333$ and $\rho(6,4) \geq \frac{29}{8}=3.625$. These two graphs are circulant graphs, which are Cayley graphs over $\mathbb{Z}_{n}$.

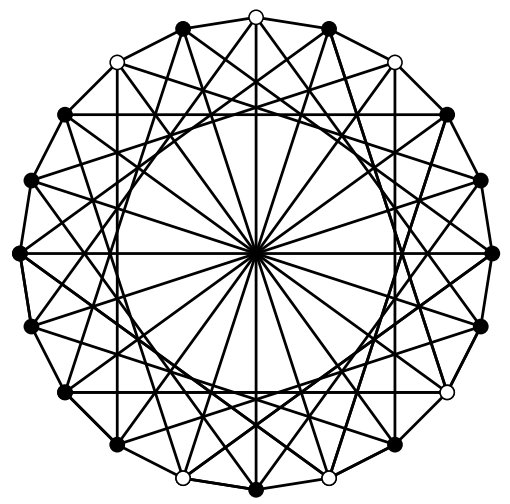

(A) A 5-regular triangle-free (vertex-transitive) graph with Hall ratio $\frac{10}{3}$. It is the Cayley graph over $\mathbb{Z}_{20}$ with generating set $\{ \pm 1, \pm 6, \pm 10\}$. There is no independent set of order 7 , and the white vertices form a independent set of order 6 .

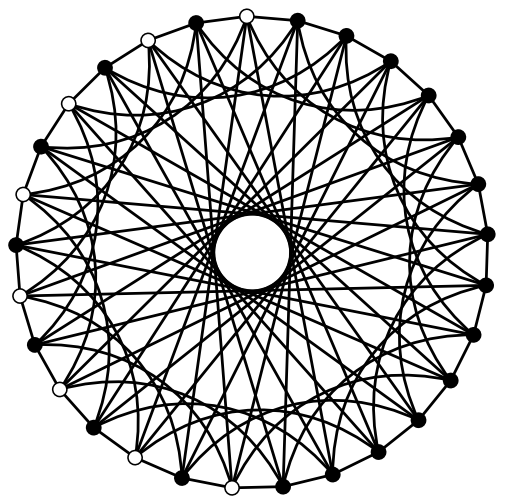

(в) A 6-regular triangle-free (vertex-transitive) graph with Hall ratio $\frac{29}{8}$. It is the Cayley graph over $\mathbb{Z}_{29}$ with generating set $\{ \pm 1, \pm 5, \pm 13\}$. There is no independent set of order 9 , and the white vertices form a independent set of order 8.

Figure 3. Two possibly extremal regular triangle-free graphs for the Hall ratio.

The value of $\rho(3, g)$ has also been studied when $g$ goes to infinity. Kardoš, Král' and Volec [15] proved the existence of an integer $g_{0}$ such that $\rho\left(3, g_{0}\right) \leq 2.2978$. More strongly, their upper bound 
holds for the fractional chromatic number of every (sub)cubic graph of girth at least $g_{0}$. In the other direction, Bollobás [4] proved a general lower bound on $\rho(d, g)$.

Theorem 5 (Bollobás, 1981). Let $d \geq 3$. Let $\alpha$ be a real number in $(0,1)$ such that

$$
\alpha(d \ln 2-\ln (\alpha))+(2-\alpha)(d-1) \ln (2-\alpha)+(\alpha-1) d \ln (1-\alpha)<2(d-1) \ln 2 .
$$

For every positive integer $g$, there exists a d-regular graph with girth at least $g$ and Hall ratio more than $2 / \alpha$.

Theorem 5 allows us to compute lower bounds on $\rho(d, \infty)$ for any value of $d$, the smaller ones being represented in Table 2. All these values can be generalised into a looser but asymptotically equivalent general lower bound of $d /(2 \ln d)$ [4, Corollary 3].

\begin{tabular}{cc}
\hline$d$ & lower bound on $\rho(d, \infty)$ \\
\hline 2 & 2 \\
3 & 2.17835 \\
4 & 2.3775 \\
5 & 2.57278 \\
6 & 2.76222 \\
7 & 2.94606 \\
8 & 3.1249 \\
9 & 3.29931 \\
10 & 3.46981 \\
$d$ & $d /(2 \ln d)$ \\
\hline
\end{tabular}

TABLE 2. Lower bounds on $\rho(d, \infty)$ implied by Theorem 5 .

1.4. Previous results on the fractional chromatic number. Recently, Molloy [18] proved the best known extremal upper bounds for the chromatic number of graphs of given clique number and maximum degree.

Theorem 6 (Molloy, 2019). Let $G$ be a graph of maximum degree $\Delta$.

- If $G$ is triangle-free, then for every $\varepsilon>0$, there exists $\Delta_{\varepsilon}$ such that, assuming that $\Delta \geq \Delta_{\varepsilon}$,

$$
\chi(G) \leq(1+\varepsilon) \frac{\Delta}{\ln \Delta} .
$$

- If $G$ has clique number $\omega(G)>2$, then

$$
\chi(G) \leq 200 \omega(G) \frac{\Delta \ln \ln \Delta}{\ln \Delta} .
$$

The first bound is sharp up to a multiplicative factor in a strong sense, since as shown by Bollobás [4, Corollaries 3 and 4] for all integers $g$ and $\Delta \geq 3$ there exists a graph with maximum degree $\Delta$, girth at least $g$ and chromatic number at least $\frac{\Delta}{2 \ln \Delta}$.

There remains however a substantial range of degrees not concerned by the bound for trianglefree graphs given by Theorem 6, namely when $\Delta$ is smaller than $\Delta_{\varepsilon}$, which is larger than $20^{2 / \varepsilon}$. Determining the maximum value of $\chi_{f}$ among triangle-free graphs of maximum degree 3 has been a long standing open problem, before it was settled in a work involving the second author [9]. They showed that it equals $\rho(3,4)$, namely $14 / 5$. The same question for larger values of the maximum degree is still open; for graphs of maximum degree 4 that value lies between 3.25 and 3.5. To this date, the best known upper bound in terms of clique number and maximum degree (when those 
two parameters are not too far apart) for the fractional chromatic number ${ }^{1}$ is due to Molloy and Reed [19, Theorem 21.7, p. 244].

Theorem 7 (Molloy and Reed, 2002). For every graph $G$,

$$
\chi_{f}(G) \leq \frac{\omega(G)+\Delta(G)+1}{2} .
$$

If one considers a convex combination of the clique number and the maximum degree plus one for an upper bound on the (fractional) chromatic number of a graph, then because the chromatic number of a graph never exceeds its maximum degree plus one, the aim is to maximise the coefficient in front of the clique number. The convex combination provided by Theorem 7 (which is conjectured to hold, after taking the ceiling, also for the chromatic number), is best possible. Indeed, for every positive integer $k$ the graph $G_{k}:=C_{5} \otimes K_{k}$ is such that $\omega\left(G_{k}\right)=2 k, \Delta\left(G_{k}\right)=3 k-1, \chi_{f}\left(G_{k}\right)=$ $\frac{5 k}{2}=\frac{\omega\left(G_{k}\right)+\Delta\left(G_{k}\right)+1}{2}$.

A local form of Theorem 7 exists: it was first devised by McDiarmid (unpublished) and appears as an exercise in Molloy and Reed's book [19]. A published version is found in the thesis of Andrew King [16, Theorem 2.10, p. 12].

Theorem 8 (McDiarmid, unpublished). Let $G$ be a graph, and set $f_{G}(v):=\frac{\omega_{G}(v)+\operatorname{deg}_{G}(v)+1}{2}$ for every $v \in V(G)$, where $\omega_{G}(v)$ is the order of a largest clique in $G$ containing $v$. Then

$$
\chi_{f}(G) \leq \max \left\{f_{G}(v): v \in V(G)\right\} .
$$

In Subsection 3.1, we slightly strengthen the local property of Theorem 8 as a way to illustrate the arguments used later on.

1.5. Our results. Our first contribution is to establish a (non-explicit) formula for an upper bound on the fractional chromatic number of triangle-free graphs depending on their maximum degree $\Delta$. The upper bound which can be effectively computed from this formula improves on the one which can be derived from Theorem 8 as soon as $\Delta \geq 17$.

\begin{tabular}{cccc}
\hline$\Delta(G)$ & $k$ & $\lambda$ & upper bound on $\chi_{f}(G)$ \\
\hline $1 \ldots 16$ & 2 & $\infty$ & $\frac{\Delta(G)+3}{2}$ \\
17 & 3 & 3.41613 & 9.91552 \\
18 & 3 & 3.50195 & 10.3075 \\
19 & 3 & 3.58603 & 10.6981 \\
20 & 3 & 3.66847 & 11.0875 \\
50 & 4 & 2.04455 & 22.1644 \\
100 & 5 & 1.48418 & 38.0697 \\
200 & 6 & 1.24061 & 66.151 \\
500 & 8 & 0.915598 & 139.842 \\
1000 & 10 & 0.734978 & 249.058 \\
\hline
\end{tabular}

TABLE 3. Upper bounds on $\chi_{f}(G)$ when $G$ is triangle-free.

\footnotetext{
${ }_{1}$ For the chromatic number, the reader is referred to a nice theorem of Kostochka [17, which for instance implies that every graph with maximum degree at most 5 and girth at least 35 has chromatic number at most 4 (Corollary 2 in loc. cit.). The general upper bound on the chromatic number guaranteed by Kostochka's theorem is never less than the floor of half the maximum degree plus two.
} 
Theorem 9. For every triangle-free graph $G$ of maximum degree $\Delta$,

$$
\chi_{f}(G) \leq 1+\min _{k \in \mathbb{N}} \inf _{\lambda>0} \frac{(1+\lambda)^{k}+\lambda(1+\lambda) \Delta}{\lambda(1+k \lambda)} .
$$

Theorem 9 lets us derive the upper bounds for the fractional chromatic number of triangle-free graphs in Table 3 . We note that considering the couple $(k, \lambda)=(2, \infty)$, Theorem 9 implies the fractional Reed bound of Theorem 7 . We also obtain the following bound as a corollary.

Corollary 1. For every triangle-free graph $G$ of maximum degree $\Delta \geq 2$,

$$
\chi_{f}(G) \leq 1+\left(1+\frac{2}{\ln \Delta}\right) \frac{\Delta}{\ln \Delta-2 \ln \ln \Delta} .
$$

Proof. The function $f: \Delta \mapsto 1+\left(1+\frac{2}{\ln \Delta}\right) \frac{\Delta}{\ln \Delta-2 \ln \ln \Delta}$ is bounded from below by 4 when $\Delta>0$, so we may assume that $\Delta \geq 4$, using the naive upper bound $\Delta+1$ for smaller values of $\Delta$.

It remains to apply Theorem 9 and consider the couple $(k, \lambda)=\left(\lfloor\ln \Delta(\ln \Delta-2 \ln \ln \Delta)\rfloor, \frac{1}{\ln \Delta}\right)$, by noting that we then have $1+k \lambda \geq \ln \Delta-2 \ln \ln \Delta$.

We note that Corollary 1 in particular implies the fractional version of the triangle-free bound of Theorem 6. Therefore Theorem 9 yields a smooth transition for the fractional chromatic number of triangle-free graphs from Reed's bound to Molloy's bound, as $\Delta$ increases.

In order to obtain upper bounds smaller than that of Theorem 8 for smaller values of the maximum degree, we need to consider graphs of higher girth. Our second contribution is to establish good upper bounds for the fractional chromatic number of graphs of girth 7. Moreover, these bounds have the same local property as those of Theorem 8 .

Theorem 10. Let $f(x):=1+\min _{k \in \mathbb{N}} \frac{2 x+2^{k-3}}{k}$. If $G$ is a graph of girth at least 7 , then $G$ admits a fractional colouring such that for every induced subgraph $H$ of $G$, the restriction of $c$ to $H$ has weight at most $f\left(\max \left\{\operatorname{deg}_{G}(v): v \in V(H)\right\}\right)$. In particular,

$$
\chi_{f}(G) \leq f(\Delta(G)) .
$$

Remark 1. In Theorem 10, if $x \geq 3$ then the minimum of the function $k \rightarrow \frac{2 x+2^{k-3}}{k}$ (over $\mathbb{N}$ ) is attained for $k=\left\lfloor 4+\log _{2} x-\log _{2} \log _{2} x\right\rceil$. So if $x \geq 3$, then $f(x)=(2 \ln 2+o(1)) x / \ln x$, which is off by a multiplicative factor $2 \ln 2$ from the asymptotic value for triangle-free graphs which can be derived from Theorem 9. The turning point happens when the maximum degree is approximately $3 \cdot 10^{6}$. We also note that for every non-negative integer $x$, the minimum of the function $k \rightarrow \frac{2 x+2^{k-3}}{k}$ (over $\mathbb{N}$ ) is attained at an integer greater than 3 .

Finally, we provide improved upper bounds on the Hall ratio of graphs of maximum degree in $\{3,4,5\}$ and girth in $\{6, \ldots, 12\}$. In particular, these are upper bounds on the fractional chromatic number of vertex-transitive graphs in these classes. These upper bounds are obtained via a systematic computer-assisted method.

Theorem 11. The values presented in Table 4 are upper bounds on $\rho(d, g)$ for $d \in\{3,4,5\}$ and $g \in$ $\{6, \ldots, 12\}$.

The bounds provided by Theorem 11 when $d \in\{3,4\}$ and $g=7$ are the same as those for $g=6$. It seems that this could be a general phenomenon. We therefore offer the following conjecture, implicitly revealing that we expect our method to produce an upper bound of 2.5 on $\rho(3,13)$.

Conjecture 1. The values presented in Table 5 are upper bounds on $\rho(d, g)$ for $d \in\{3,4,5\}$ and $g \in\{6,8,10,12\}$. 


\begin{tabular}{|c|c|c|c|c|c|c|c|}
\hline . & 6 & 7 & 8 & 9 & 10 & 11 & 12 \\
\hline 3 & $30 / 11 \approx 2.727272$ & $30 / 11$ & 2.625224 & 2.604167 & 2.557176 & 2.539132 & 2.510378 \\
\hline 4 & $41 / 13 \approx 3.153846$ & $41 / 13$ & 3.038497 & 3.017382 & 3 & & \\
\hline 5 & $69 / 19 \approx 3.631579$ & 3.6 & 3.5 & & & & \\
\hline
\end{tabular}

TABLE 4. Upper bounds on $\rho(d, g)$ for $d \in\{3,4,5\}$ and $g \in\{6, \ldots, 12\}$.

\begin{tabular}{ccccc}
\hline$g$ & 6 & 8 & 10 & 12 \\
\hline 3 & & 2.604167 & 2.539132 & 2.5 \\
4 & & 3.017382 & 3 & \\
5 & 3.6 & 3.5 & & \\
\hline
\end{tabular}

TABle 5. Conjectured upper bounds on $\rho(d, g)$ for $d \in\{3,4,5\}$ and $g \in\{6, \ldots, 12\}$.

1.6. Notations. We introduce some notations before establishing a few technical lemmas, from which we will prove Theorems 10 and 11. If $v$ is a vertex of a graph $G$ and $r$ a non-negative integer, then $N_{G}^{r}(v)$ is the set of all vertices of $G$ at distance exactly $r$ from $v$ in $G$, while $N_{G}^{r}[v]$ is $\bigcup_{j=0}^{r} N_{G}^{j}(v)$. If $u$ is also a vertex of $G$, we write $\operatorname{dist}_{G}(u, v)$ for the distance in $G$ between $u$ and $v$. Further, if $J$ is a subset of vertices of $G$, then we write $N_{G}(J)$ for the set of vertices that are not in $J$ and have a neighbour in $J$, while $N_{G}[J]$ is $N_{G}(J) \cup J$. We will omit the graph subscript when there is no ambiguity, and sometimes write $N_{X}(v)$ instead of $N(v) \cap X$, for any subset of vertices $X \subseteq V(G)$. The set of all independent sets of $G$ is $\mathscr{I}(G)$, while $\mathscr{I}_{\max }(G)$ is the set of all maximal independent sets of $G$ and $\mathscr{I}_{\alpha}(G)$ is the set of all maximum independent sets of $G$. If $w$ is a mapping from $\mathscr{I}(G)$ to $\mathbb{R}$ then for every vertex $v \in V(G)$ we set

$$
w[v]:=\sum_{\substack{I \in \mathscr{I}(G) \\ v \in I}} w(I) .
$$

Further, if $\mathscr{I}$ is a collection of independent sets of $G$, then $w(\mathscr{I}):=\sum_{I \in \mathscr{I}} w(I)$. If $I$ in a independent set of a graph $G$, a vertex $v$ is covered by $I$ if $v$ belongs to $I$ or has a neighbour in $I$. A vertex that is not covered by $I$ is uncovered (by $I$ ). If $G$ is a graph rooted at a vertex $v$, then for every positive integer $d$, the set of all vertices at distance $d$ from $v$ in $G$ is a layer of $G$.

\section{TEChNiCAL Lemmas}

In this section we present the tools needed for the proofs of the main theorems.

2.1. Greedy fractional colouring algorithm. Our results on fractional colouring are obtained using a greedy algorithm analysed in a recent work involving the first author $[8$. This algorithm is a generalisation of an algorithm first described in the book of Molloy and Reed [19, p. 245] for the uniform distribution over maximum independent sets. The setting here is, for each induced subgraph $H$ of the graph we wish to fractionally colour, a probability distribution over the independent sets of $H$. We shall use only distributions over maximal independent sets.

Lemma 1 (de Joannis de Verclos et al., 2018). Let $G$ be a graph given with well-chosen parameters $\alpha_{v}, \beta_{v}$ for every vertex $v \in V(G)$. For every induced subgraph $H$ of $G$, let $\mathbf{I}_{H}$ be a random 
independent set of $H$ drawn according to a given probability distribution, and assume that

$$
\alpha_{v} \mathbb{P}\left[v \in \mathbf{I}_{H}\right]+\beta_{v} \mathbb{E}\left[\left|N(v) \cap \mathbf{I}_{H}\right|\right] \geq 1,
$$

for every vertex $v \in V(H)$. Then the greedy fractional colouring algorithm defined by Algorithm 1 produces a fractional colouring $w$ of $G$ such that the restriction of $w$ to any subgraph $H$ of $G$ is a fractional colouring of $H$ of weight at most $\max _{v \in V(H)} \alpha_{v}+\beta_{v} \operatorname{deg}_{G}(v)$. In particular,

$$
\chi_{f}(G) \leq \max _{v \in V(G)} \alpha_{v}+\beta_{v} \operatorname{deg}_{G}(v) .
$$

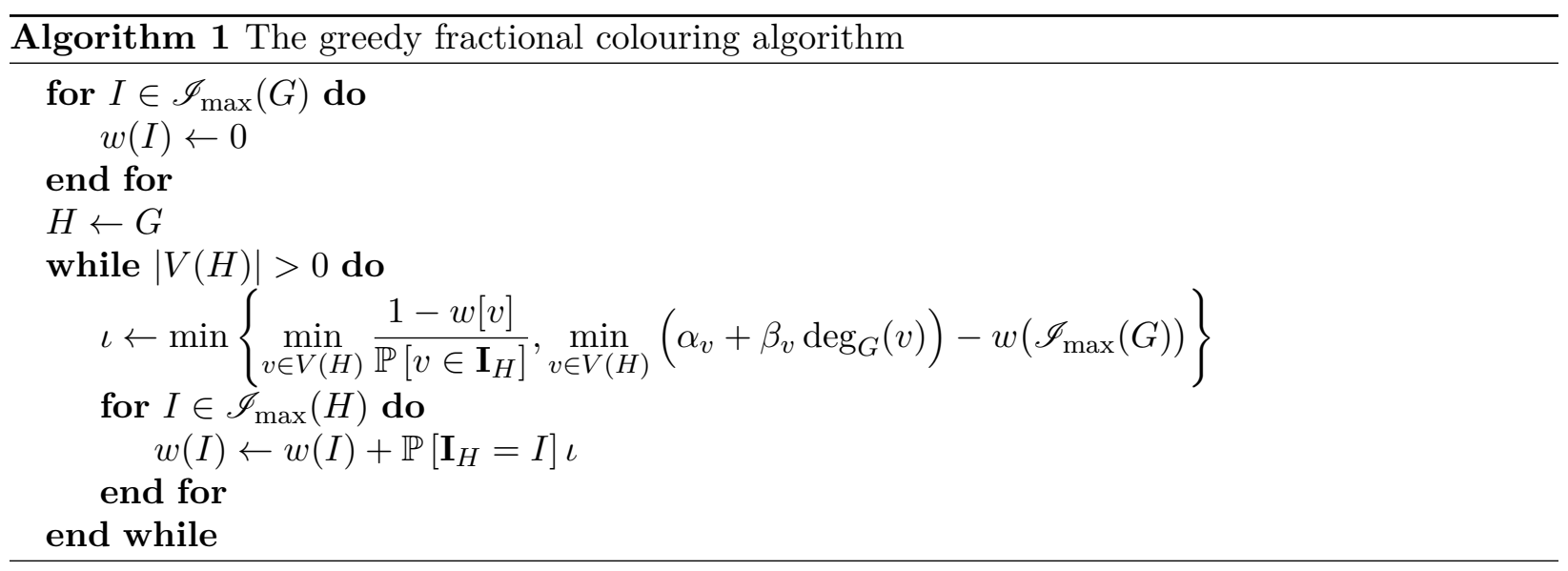

2.2. Hard-core model. In the setting of Lemma 1, we need a probability distribution over the independent sets of a given graph $H$. For instance, Molloy and Reed used the uniform distribution over the maximum independent sets of $H$, and obtained the fractional Reed bound as a result. As we will show in Section 3.1 this bound is best possible when restricting to the maximum independent sets, even for trees. Therefore, we need to include the non-maximum independent sets with non-zero probability in order to hope for improved bounds. Moreover, in order to perform a local analysis of the possible random outcomes, we need our probability distribution to have a good relative independence between the random outcomes in a local part of the graph, and the ones outside this part.

The probability distribution that we are going to use as a setting of Lemma 1 is the hard-core distribution over the independent sets of a graph, which has the Spatial Markov Property. Given a family $\mathscr{I}$ of independent sets of a graph $H$, and a positive real $\lambda$, a random independent set $\mathbf{I}$ drawn according to the hard-core distribution at fugacity $\lambda$ over $\mathscr{I}$ is such that

$$
\mathbb{P}[\mathbf{I}=I]=\frac{\lambda^{|I|}}{\sum_{J \in \mathscr{I}} \lambda^{|J|}},
$$

for every $I \in \mathscr{I}$.

Along this work, we consider two possible families $\mathscr{I}$ of independent sets of $H$, the first one being the whole set $\mathscr{I}(H)$ of independent sets of $H$. Note that when $\mathscr{I}=\mathscr{I}(H)$, and $\lambda \rightarrow \infty$, the hard-core distribution converges towards the uniform distribution over the maximum independent sets of $H$.

Lemma 2 (Spatial Markov Property). Given a graph $H$, and a real $\lambda>0$, let $\mathbf{I}$ be drawn according to the hard-core distribution at fugacity $\lambda$ over the independent sets $\mathscr{I}(H)$ of $H$. Let $X \subseteq V(H)$ be any given subset of vertices, and $J$ any possible outcome of $\mathbf{I} \backslash X$. Then, conditioned on the fact 
that $\mathbf{I} \backslash X=J$, the random independent set $\mathbf{I} \cap X$ follows the hard-core distribution at fugacity $\lambda$ over the independent sets of $H[X \backslash N(J)]$.

The proof of this result is standard and follows from a simple consideration of the marginal probabilities. Things are more complicated with our second choice for $\mathscr{I}$, that is the set $\mathscr{I}_{\max }(H)$ of maximal independent sets of $H$. Indeed, in this setting, one has to make sure that the local outcome of the independent set is compatible with the fact that the global outcome of the independent set is maximal, i.e. there remains no uncovered vertices in $H$. This adds a new level of dependency, and we need the extra assumption ( $\star$ ) to be able to handle it. For two disjoint subsets of vertices $X$ and $U$ of a graph $G$, we define $P_{X}^{2}(U)$ to be the set of vertices $x \in X$ such that there exists a path $u x^{\prime} x$ of length 2 with $u \in U$ and $x^{\prime} \in X$.

Lemma 3 (Spatial Markov Property for maximal independent sets). Given a graph $H$, and a real $\lambda>0$, let $\mathbf{I}$ be drawn according to the hard-core distribution at fugacity $\lambda$ over the maximal independent sets $\mathscr{I}_{\max }(H)$ of $H$. Let $X \subseteq V(H)$ be any given subset of vertices, $J$ any possible outcome of $\mathbf{I} \backslash X$, and $U:=(V(H) \backslash X) \backslash N[J]$ the set of vertices outside of $X$ that are uncovered $($ by $J)$. Moreover, we assume that

$$
|N(v) \cap X| \leq 1,
$$

for any vertex $v \notin X$. Then, conditioned on the fact that $\mathbf{I} \backslash X=J$, the random independent set $\mathbf{I} \cap X$ follows the hard-core distribution at fugacity $\lambda$ over the maximal independent sets of

$$
H\left[X \backslash\left(N(J) \cup P_{X}^{2}(U)\right] .\right.
$$

Proof. Set $W:=X \backslash\left(N(J) \cup P_{X}^{2}(U)\right)$. First let $I_{X}$ be any possible realisation of $\mathbf{I} \cap X$, conditioned on the fact that $\mathbf{I} \backslash X=J$. We prove that $I_{X} \in \mathscr{I}_{\max }(H[W])$. To this end, we begin by showing that $N_{X}(U) \subseteq I_{X}$. By the definitions of $J$ and $U$, every vertex in $U$ must be adjacent to a vertex in $X$, and hence for each $u \in U$ there exists a unique vertex $v_{u}$ in $X$ that is adjacent to $U$. It follows that $N_{X}(U)$ is contained in $I_{X}$. This in particular implies that no vertex in $P_{X}^{2}(U)$ can belong to $I_{X}$, and hence $I_{X} \subseteq W$. We note for later that we just established that $N_{X}(U)$ is a set of isolated vertices of $H[W]$ (that is, these vertices belong to $W$ and have no neighbour in $H[W]$ ).

Next we observe that $I_{X}$ is maximal in $H[W]$. Indeed, let $w \in W \backslash I_{X}$. Because $I_{X} \cup J$ is a maximal independent set of $H$, there exists $v \in I_{X} \cup J$ that is adjacent to $w$ in $H$. Since $W \subseteq X \backslash N(J)$ by definition, we deduce that $v \in I_{X}$ and hence $I_{X}$ is maximal in $H[W]$.

Second, given any set $I_{X} \in \mathscr{I}_{\max }(H[W])$, the set $I_{X} \cup J$ is a valid realisation of $\mathbf{I}$. Indeed, $I_{X}$ and $J$ are independent sets, and so is their union as $I_{X} \cap N(J)=\varnothing$. To prove that $I_{X} \cup J$ is maximal in $H$, it suffices to show that every vertex $x$ in $U \cup(X \backslash W)$ has a neighbour in $I_{X}$. As reported earlier, $N_{X}(U)$ is contained in $W$ and forms a set of isolated vertices in $H[W]$. Therefore, $N_{X}(U)$ is contained in every maximal independent of $H[W]$, and hence in $U_{X}$. Since every vertex in $U$ has a neighbour in $X$, it therefore only remains to deal with case where $x \in X \backslash W$. Then $x \in N(J) \cup P_{X}^{2}(U)$, and hence $x$ has a neighbour in $J \cup N_{X}(U)$, which is contained in $I_{X} \cup J$.

In conclusion, the set of realisations of $\mathbf{I} \cap X$ is exactly $\mathscr{I}_{\max }(H[W])$, and each such realisation $I_{X}$ has a probability proportional to $\lambda^{\left|I_{X}\right|+|J|}$, and hence proportional to $\lambda^{\left|I_{X}\right|}$ since $J$ is fixed. This finishes the proof.

2.3. Independence ratio. We state two lemmas which can be proved in similar ways. We only present the proof of the second one, the argument for the first lemma being very close but a little simpler.

Lemma 4. Let $r$ be a positive integer and $G$ be a d-regular graph on $n$ vertices. Let $\alpha_{0}, \ldots, \alpha_{r}$ be real numbers such that $\sum_{i=1}^{r} \alpha_{i}(d-1)^{i-1} \geq 0$. Assume that there exists a probability distribution $p$ 
on $\mathscr{I}_{\max }(G)$ such that

$$
\forall v \in V(G), \quad \sum_{i=0}^{r} \alpha_{i} \mathbb{E}\left[\mathbf{X}_{i}(v)\right] \geq 1,
$$

where $\mathbf{X}_{i}(v)$ is the random variable counting the number of paths of length $i$ between $v$ and a vertex belonging to a random independent set $\mathbf{I}$ chosen following $p$. Then

$$
\frac{n}{\alpha(G)} \leq \alpha_{0}+\sum_{i=1}^{r} \alpha_{i} d(d-1)^{i-1}
$$

Lemma 5. Let $r$ be a positive integer and $G$ be a d-regular graph on $n$ vertices. Let $\alpha_{0}, \ldots, \alpha_{r}$ be real numbers such that $\sum_{i=0}^{r} \alpha_{i}(d-1)^{i} \geq 0$. Assume that there exists a probability distribution $p$ on $\mathscr{I}_{\max }(G)$ such that

$$
\forall e \in E(G), \quad \sum_{i=0}^{r} \alpha_{i} \mathbb{E}\left[\mathbf{X}_{i}(e)\right] \geq 1,
$$

where $\mathbf{X}_{i}(e)$ is the random variable counting the number of paths of length $i+1$ starting with $e$ and ending at a vertex belonging to a random independent set $\mathbf{I}$ chosen following $p$. Then

$$
\frac{n}{\alpha(G)} \leq \sum_{i=0}^{r} 2 \alpha_{i}(d-1)^{i}
$$

Proof. Given an integer $i \in\{0, \ldots, r\}$ and an edge $e$ of $G$, the contribution of an arbitrary vertex $v \in$ $\mathbf{I}$ to $\mathbf{X}_{i}(e)$ is the number of paths of length $i+1$ starting at $v$ and ending with $e$. It follows that the total contribution of any vertex $v \in \mathbf{I}$ to $\sum_{e \in E(G)} \mathbf{X}_{i}(e)$ is the number of paths of $G$ with length $i+1$ that start at $v$, which is $d(d-1)^{i}$ since $G$ is a $d$-regular graph. Consequently,

$$
\mathbb{E}\left[\sum_{e \in E(G)} \mathbf{X}_{i}(e)\right]=\sum_{v \in V(G)} \mathbb{P}[v \in \mathbf{I}] d(d-1)^{i} .
$$

We now sum (3) over all edges of $G$.

$$
\begin{aligned}
\sum_{e \in E(G)} \sum_{i=0}^{r} \alpha_{i} \mathbb{E}\left[\mathbf{X}_{i}(e)\right] & \geq|E(G)|=\frac{n d}{2} \\
\sum_{i=0}^{r} \alpha_{i} \sum_{e \in E(G)} \mathbb{E}\left[\mathbf{X}_{i}(e)\right] & \geq \frac{n d}{2} \\
\sum_{i=0}^{r} \alpha_{i} \sum_{v \in V(G)} \mathbb{P}[v \in \mathbf{I}] d(d-1)^{i} & \geq \frac{n d}{2} \\
\sum_{i=0}^{r} 2 \alpha_{i} \mathbb{E}[|\mathbf{I}|](d-1)^{i} & \geq n \\
\sum_{i=0}^{r} 2 \alpha_{i}(d-1)^{i} & \geq \frac{n}{\alpha(G)}
\end{aligned}
$$

The next lemma allows us to generalise Lemmas 4 and 5 to non-regular graphs. To this end, we use a standard argument coupled with the existence of specific vertex-transitive type-1 regular graphs with any given degree and girth. These are provided by a construction of Exoo and Jajcay [12] in the proof of their Theorem 19, which is a direct generalisation of a construction for cubic 
graphs designed by Biggs [3, Theorem 6.2]. We slighty reformulate their theorem, the mentioned edge-colouring and transitivity property following simply from the fact that the graph constructed is a Cayley graph obtained from a generating set consisting only of involutions. Given a graph $G$ endowed with an edge-colouring $c$, an automorphism $f$ of $G$ is c-preserving if $c(\{f(u), f(v)\})=c(u, v)$ for each edge $\{u, v\}$ of $G$. The graph $G$ is $c$-transitive if for every pair $(u, v)$ of vertices of $G$ there exists a $c$-preserving automorphism $f$ of $G$ such that $f(u)=v$.

Theorem 12 (Exoo \& Jajcay, 2013). For every integers $d$ and $g$ both at least 3, there exists a $d$-regular graph $H$ with girth at least $g$ along with a proper edge-colouring $c$ using $d$ colours such that $H$ is c-transitive.

Lemma 6. From any graph $G$ of maximum degree $d$ and girth $g$, we can construct a d-regular graph $\varphi(G)$ of girth $g$ whose vertex set can be partitioned into induced copies of $G$, and such that any vertex $v \in G$ can be sent to any of its copies through an automorphism.

Proof. Set $k:=\sum_{v \in G}(d-\operatorname{deg}(v))$. Let $G^{\prime}$ be the supergraph of $G$ obtained by adding $k$ vertices $v_{1}^{\prime}, \ldots, v_{k}^{\prime}$ each of degree 1 , such that all other vertices have degree $d$. We let $e_{i}^{\prime}$ be the edge of $G^{\prime}$ incident to $v_{i}^{\prime}$, for each $i \in\{1, \ldots, k\}$. By Theorem 12, there exists a $k$-regular graph $H$ of girth at least $g$ together with a proper edge-colouring $c$ using $k$ colours, such that $H$ is $c$-transitive. Let $n(H)$ be the number of vertices of $H$ and write $V(H)=\{1, \ldots, n(H)\}$.

We construct $\varphi(G)$ by starting from the disjoint union of $n(H)$ copies $G_{1}, \ldots, G_{n(H)}$ of $G$. For each edge $e=\{i, j\} \in E(H)$, letting $u_{e}$ be the vertex of $G$ incident to the edge $e_{c(e)}^{\prime}$ in $G^{\prime}$, we add an edge between the copy of $u_{e}$ in $G_{i}$ and that in $G_{j}$.

Any cycle in $\varphi(G)$ either is a cycle in $G$, and hence has length at least $g$, or contains all the edges of a cycle in $H$, and hence has length at least $g$. It follows that $\varphi(G)$ has girth $g$.

The last statement follows directly from the fact that $H$ is $c$-transitive.

Corollary 2. Let $d$ and $g$ be integers greater than two. If there exists a constant $B=B(d, g)$ such that every $d$-regular graph $H$ with girth $g$ has independence ratio at least $B$, then every graph $G$ with maximum degree $d$ and girth $g$ also has independence ratio at least B. In particular, if Lemma 4 or Lemma 5 can be applied to the class of d-regular graphs of girth $g$, then the conclusion also holds for the class of graphs with maximum degree $d$ and girth $g$, that is, for $\rho(d, g)$.

Proof. Let $G$ be a graph with maximum degree $d$ and girth $g$ on $n$ vertices. Let $\varphi(G)$ be the graph provided by Lemma 6 . In particular, $|V(\varphi(G))|=k n$ where $k$ is the number of induced copies of $G$ partitioning $V(\varphi(G))$. By assumptions, $\varphi(G)$ contains a independent set $I$ of order at least $B \cdot k n$. Letting $I_{i}$ be the set of vertices of the $i$-th copy of $G$ contained in $I$, by the pigeon-hole principle there exists $i \in\{1, \ldots, k\}$ such that $\left|I_{i}\right| \geq B \cdot n$, and hence $G$ has independence ratio at least $B$.

\section{Fractional Colourings}

3.1. A local version of Reed's bound. For the sake of illustration, we begin by showing how Lemma 1 can be used to prove Theorem 7 . We actually establish a slight strengthening of Theorem 8, the local form of Theorem 7. The argument relies on the relation (A) below [16, Lemma 2.11], which is a local version of the relation (21.10) appearing in Molloy and Reed's book [19]. The short argument, however, stays the same and we provide it here only for explanatory purposes, since it is the inspiration for the argument used in the proof of Theorem 10.

Proposition 1. Let $G$ be a graph, and set $f_{G}(v):=\frac{\omega_{G}(v)+\operatorname{deg}_{G}(v)+1}{2}$ for every $v \in V(G)$, where $\omega_{G}(v)$ is the order of a largest clique in $G$ containing $v$. Then $G$ admits a fractional colouring $c$ such that the restriction of $c$ to any induced subgraph $H$ of $G$ has weight at most $\max _{v \in V(H)} f_{G}(v)$. In particular,

$$
\chi_{f}(G) \leq \max \left\{f_{G}(v): v \in V(G)\right\} .
$$


Proof. We demonstrate the statement by applying Lemma 1. To this end, we use the uniform distribution on maximum independent sets, which corresponds to the hard-core distribution at fugacity $\lambda=\infty$.

(A). For every induced subgraph $H$ of $G$, let $\mathbf{I}_{H}$ be a maximum independent set of $H$, drawn uniformly at random. Then for every vertex $v \in V(H)$,

$$
\frac{\omega(v)+1}{2} \mathbb{P}\left[v \in \mathbf{I}_{H}\right]+\frac{1}{2} \mathbb{E}\left[\left|N(v) \cap \mathbf{I}_{H}\right|\right] \geq 1 .
$$

The conclusion then follows by applying Lemma 1, with $r=1, \alpha_{v}=\frac{1}{2} \cdot(\omega(v)+1)$ and $\beta_{v}=\frac{1}{2}$ for every vertex $v \in V(G)$.

It remains to establish (A). We let $J$ be any possible outcome of $\mathbf{I}_{H} \backslash N[v]$, and $W=N[v] \backslash N(J)$. We condition on the random event $E_{J}$ that $\mathbf{I}_{H} \backslash N[v]=J$, and the Spatial Markov Property of the uniform distribution over the maximum independent sets of $H$ ensures that $\mathbf{I}_{H} \cap W$ is a uniform random maximum independent set of $H[W]$. There are two cases.

(i) If $W$ is a clique of size $k \leq \omega(v)$, then exactly one vertex from $W$ belongs to $\mathbf{I}_{H}$, and every vertex in $W$ has equal probability $1 / k$ to be in $\mathbf{I}_{H}$. So, in this case,

$$
\frac{\omega(v)+1}{2} \mathbb{P}\left[v \in \mathbf{I}_{H} \mid E_{J}\right]+\frac{1}{2} \mathbb{E}\left[\left|N(v) \cap \mathbf{I}_{H}\right| \mid E_{J}\right]=\frac{\omega(v)+1}{2 k}+\frac{k-1}{2 k} \geq 1 .
$$

(ii) If $W$ is not a clique, then $\left|W \backslash\{v\} \cap \mathbf{I}_{H}\right| \geq 2$ and $v \notin \mathbf{I}_{H}$, since $\mathbf{I}_{H}$ is a maximum independent set. So, in this case,

$$
\frac{\omega(v)+1}{2} \mathbb{P}\left[v \in \mathbf{I}_{H} \mid E_{J}\right]+\frac{1}{2} \mathbb{E}\left[\left|N(v) \cap \mathbf{I}_{H}\right| \mid E_{J}\right] \geq \frac{1}{2} \times 2=1
$$

The validity of A follows by summing over all possible realisations $J$ of $\mathbf{I}_{H} \backslash N[v]$.

We finish by noting that the bound provided by Theorem 8 is best possible over the class of unicyclic triangle-free graphs if one uses the fractional greedy colouring of Lemma 1 together with any probability distribution on the maximum independent sets of the graph.

Lemma 7. If the probability distribution used in Lemma 1 gives positive probability only to maximum independent sets, then the greedy fractional colouring algorithm can return a fractional colouring of weight up to $\frac{d+3}{2}$ in general for graphs of degree $d$, should they be acyclic when $d$ is odd, or have a unique cycle (of length 5) when $d$ is even.

Proof. We prove the statement by induction on the positive integer $d$.

- If $d=1$, then let $G_{1}$ consist only of an edge. The algorithm returns a fractional colouring of $G_{1}$ of weight 2 .

- If $d=2$, then let $G_{2}$ be the cycle of length 5 . The algorithm returns a fractional colouring of $G_{2}$ of weight $\frac{5}{2}$.

- If $d>2$, then let $G_{d}$ be obtained from $G_{d-2}$ by adding two neighbours of degree 1 to every vertex. This creates no new cycles, so $G_{d}$ is acyclic when $d$ is odd, and contains a unique cycle, which is of length 5 , when $d$ is even.

For every $d \geq 3$, the graph $G_{d}$ contains a unique maximum independent set, namely $I_{0}:=$ $V\left(G_{d}\right) \backslash V\left(G_{d-2}\right)$. After the first step of the algorithm applied to $G_{d}$, all the vertices in $I_{0}$ have weight 1 , and we are left with the graph $G_{d-2}$ where every vertex has weight 0 . By the induction hypothesis, the total weight of the fractional colouring returned by the algorithm is therefore $1+\frac{(d-2)+3}{2}=\frac{d+3}{2}$. 
3.2. Triangle-free graphs. Using a similar approach, it is possible to obtain improved bounds for the fractional chromatic number of a given triangle-free graph $G$, if we apply Lemma 1 with a independent set $\mathbf{I}_{H}$ drawn according to the hard-core distribution at fugacity $\lambda$ over the set $\mathscr{I}(H)$ of all independent sets of any induced subgraph $H$ of $G$, for a carefully chosen $\lambda>0$.

(B). For every subgraph $H$ of $G$, every vertex $v \in V(H)$, and every integer $k \geq 1$,

$$
\left(1+\frac{(1+\lambda)^{k}}{\lambda(1+k \lambda)}\right) \mathbb{P}\left[v \in \mathbf{I}_{H}\right]+\frac{1+\lambda}{1+k \lambda} \mathbb{E}\left[\left|N(v) \cap \mathbf{I}_{H}\right|\right] \geq 1 .
$$

Note that when $\omega=2$, we deduce $(\mathrm{A})$ from $(\mathrm{B})$ by taking $k=2$ and letting $\lambda$ go to infinity.

Proof. We let $J$ be any possible realisation of $\mathbf{I}_{H} \backslash N[v]$. By the Spatial Markov Property of the hardcore distribution, if we condition on the event $E_{J}$ that $\mathbf{I}_{H} \backslash N[v]=J$ and write $W:=N[v] \backslash N(J)$, then $\mathbf{I}_{H} \cap N[v]$ follows the hard-core distribution at fugacity $\lambda$ over $\mathscr{I}(H[W]$ ). Since $G$ (and therefore also $H)$ is triangle-free, then $H[W]$ is a star $K_{1, d}$, for some integer $d \in\{0, \ldots, \Delta(G)\}$. An analysis of the hard-core distribution over the independent sets of a star yields that

(i) $\mathbb{P}\left[v \in \mathbf{I}_{H} \mid E_{J}\right]=\frac{\lambda}{\lambda+(1+\lambda)^{d}}$, and

(ii) $\mathbb{E}\left[\left|N(v) \cap \mathbf{I}_{H}\right| \mid E_{J}\right]=\frac{d \lambda(1+\lambda)^{d-1}}{\lambda+(1+\lambda)^{d}}$.

For some positive real numbers $\alpha$ and $\beta$, we let

$$
g(x):=\alpha \frac{\lambda}{\lambda+(1+\lambda)^{x}}+\beta \frac{x \lambda(1+\lambda)^{x-1}}{\lambda+(1+\lambda)^{x}} .
$$

We observe that $g$ is a convex function, and therefore its minimum over the (non-negative) reals is reached at its unique critical point $x^{*}$ such that $g^{\prime}\left(x^{*}\right)=0$ (if it exists). Moreover, if there are numbers $y$ and $z>y$ such that $g(y)=g(z)$, then Rolle's theorem ensures that $x^{*} \in(y, z)$, and $g(x) \geq g(y)$ for every $x \notin(y, z)$.

Let $k$ be a positive integer. We now fix

$$
\alpha:=1+\frac{(1+\lambda)^{k}}{\lambda(1+k \lambda)} \quad \text { and } \quad \beta=\frac{1+\lambda}{1+k \lambda} .
$$

One can easily check that with these values for $\alpha$ and $\beta$, it holds that

$$
g(k-1)=1 \quad \text { and } \quad g(k)=1 .
$$

We conclude that $g(d) \geq 1$ for every non-negative integer $d$, which means that

$$
\left(1+\frac{(1+\lambda)^{k}}{\lambda(1+k \lambda)}\right) \mathbb{P}\left[v \in \mathbf{I}_{H} \mid E_{J}\right]+\frac{1+\lambda}{1+k \lambda} \mathbb{E}\left[\left|N(v) \cap \mathbf{I}_{H}\right| \mid E_{J}\right] \geq 1,
$$

for any possible realisation $J$ of $\mathbf{I}_{H} \backslash N[v]$. The conclusion follows again by a convex combination of (5) over all the possible values of $J$.

3.3. A stronger bound for graphs of girth 7. Let $G$ be a graph of girth (at least) 7 and $H$ an induced subgraph of $G$. We wish to apply Lemma 1 with a independent set $\mathbf{I}_{H}$ draw according to the hard-core distribution at fugacity $\lambda$ over the set $\mathscr{I}_{\max }(H)$ of all maximal independent sets of $H$, for the specific value $\lambda=4$. We now establish the following assertion.

(C). For every given vertex $v \in V(H)$ and every integer $k \geq 4$,

$$
\frac{2^{k-3}+k}{k} \mathbb{P}\left[v \in \mathbf{I}_{H}\right]+\frac{2}{k} \mathbb{E}\left[\left|N(v) \cap \mathbf{I}_{H}\right|\right] \geq 1 .
$$


Proof. Let $J$ be any possible realisation of $\mathbf{I}_{H} \backslash N^{2}[v]$. We are going to condition on the random event $E_{J}$ that $\mathbf{I}_{H} \backslash N^{2}[v]=J$. Let $U:=\left(V(H) \backslash N^{2}[v]\right) \backslash N[J]$ be the set of vertices at distance more than 2 from $v$ that are uncovered (by $J$ ), and set $W:=N^{2}[v] \backslash\left(N(J) \cup P_{N^{2}[V]}^{2}(U)\right.$ ). (It follows from the definitions that $v \in W$.) Because the girth of $G$ is greater than 6 , no vertex outside of $N^{2}[v]$ has more than one neighbour in $N^{2}[v]$. Then Lemma 3 ensures that $\mathbf{I}_{H} \cap W$ follows the hard-core distribution at fugacity $\lambda=4$ over the maximal independent sets of $H[W]$.

In general, $H[W]$ is not connected, but it is sufficient for us to assume that it is, by considering the connected component of $H[W]$ containing $v$.

We let $W_{i}$ be the set of vertices in $W$ at distance $i$ from $v$ in $H[W]$, for $i \in\{0,1,2\}$, and $W_{1, j}$ be the subset of vertices of $W_{1}$ with $j$ neighbours in $W_{2}$. We set $x_{j}:=\left|W_{1, j}\right|$. Thus $\left|W_{1}\right|=\sum_{j=0}^{d-1} x_{j}$ and $\left|W_{2}\right|=\sum_{j=1}^{d-1} j x_{j}$, since $G$ has girth greater than 4 .

Note that $W_{1} \in \mathscr{I}_{\max }(H[W])$ and that $\mathbb{P}\left[\mathbf{I}_{H} \cap W=W_{1}\right]$ is proportional to $\lambda^{\sum_{j=0}^{d-1} x_{j}}$. In order to ease the following computations and verifications, we compute a weight $w(I)$ for each independent set $I \in \mathscr{I}_{\max }(H[W])$ that is proportional to $\mathbb{P}\left[\mathbf{I}_{H} \cap W=I\right]$, such that $w\left(W_{1}\right)=1$.

There is exactly one maximal independent set $I_{0}$ that contains $v$, namely $I_{0}:=\{v\} \cup W_{2}$, of normalised weight $w_{0}:=w\left(I_{0}\right)=\lambda^{1+\sum_{j \geq 0}(j-1) x_{j}}$. Every other maximal independent set $I \in$ $\mathscr{I}_{\max }(H[W]) \backslash\left\{I_{0}, W_{1}\right\}$ contains $W_{1,0}$. In addition, for every vertex $u \in W_{1} \backslash W_{1,0}$, the set $I$ either contains $u$ or it contains all the neighbours of $u$ in $W_{2}$. Therefore, it follows that if $x_{0}>0$, then the sum of the weights of these other independent sets is

$$
T:=\sum_{i_{1} \leq x_{1}, \ldots, i_{d-1} \leq x_{d-1}} \prod_{j=1}^{d-1}\left(\begin{array}{c}
x_{j} \\
i_{j}
\end{array}\right)\left(\lambda^{j-1}\right)^{i_{j}}=\prod_{j=1}^{d-1}\left(1+\lambda^{j-1}\right)^{x_{j}} .
$$

If $x_{0}=0$, then the sum of their weights is $T-\frac{w_{0}}{\lambda}$, since there is no independent set containing $W_{2}$ in whole and not $v$ in this case.

We let $D:=w_{0}+T$ if $x_{0}>0$, and $D:=T+w_{0}\left(1-\frac{1}{\lambda}\right)$ otherwise. It follows that

$$
\begin{aligned}
\mathbb{P}\left[v \in \mathbf{I}_{H} \mid E_{J}\right] & =\frac{w_{0}}{D} \quad \text { and } \\
\mathbb{E}\left[\left|N(v) \cap \mathbf{I}_{H}\right| \mid E_{J}\right] & =\frac{T}{D}\left(x_{0}+\sum_{j=1}^{d-1} \frac{x_{j}}{1+\lambda^{j-1}}\right) .
\end{aligned}
$$

There remains to check that, when $\lambda=4$, it holds that

This translates to

$$
\frac{2^{k-3}+k}{k} \mathbb{P}\left[v \in \mathbf{I}_{H} \mid E_{J}\right]+\frac{2}{k} \mathbb{E}\left[\left|N(v) \cap \mathbf{I}_{H}\right| \mid E_{J}\right] \geq 1 \text {. }
$$

$$
\begin{array}{rlrl}
\left(2^{k-3}+\frac{k}{\lambda}\right) \cdot w_{0} & \geq T\left(k-2 \sum_{j=1}^{d-1} \frac{x_{j}}{1+\lambda^{j-1}}\right) & & \text { if } x_{0}=0, \text { and to } \\
2^{k-3} w_{0} \geq T\left(k-2 x_{0}-2 \sum_{j=1}^{d-1} \frac{x_{j}}{1+\lambda^{j-1}}\right) & & \text { if } x_{0} \neq 0 .
\end{array}
$$

Notice that the condition is verified if $x_{0} \geq k / 2$, so we assume from now on that $x_{0}<k / 2$. We use the two following facts. 
Fact 1: For every positive integer $j$, the function $\lambda \mapsto\left(1+\frac{1}{\lambda^{j-1}}\right)^{1+\lambda^{j-1}}$ is non increasing on $(0,+\infty)$, and in particular always bounded from above by $\frac{3125}{1024}$ when $\lambda \geq 4$ and $j \geq 2$, and by $\left(1+\frac{1}{\lambda^{j_{0}-1}}\right)^{1+\lambda^{j_{0}-1}}$ when $\lambda \geq 1$ and $j \geq j_{0}$.

Fact 2: For all real numbers $y_{0}, A$ and $B$ with $A>1$ and $B>0$, the maximum of the function $f: y \mapsto A^{y}(B-2 y)$ on the domain $\left[y_{0},+\infty\right)$ is $f\left(y_{0}\right)$ when $B / 2-1 / \ln A \leq y_{0}$, and $\frac{2 A^{B / 2}}{e \ln A}$ otherwise.

Let us discriminate on the possible values for $x_{0}$, noting that $w_{0} \geq \lambda^{1-x_{0}}$.

(i) When $x_{0}=0$, it suffices to show that

$$
2^{k-3} \lambda+k \geq \prod_{j=1}^{d-1}\left(1+\frac{1}{\lambda^{j-1}}\right)^{x_{j}}\left(k-2 \sum_{j=1}^{d-1} \frac{x_{j}}{1+\lambda^{j-1}}\right) .
$$

(ii) When $1 \leq x_{0}<k / 2$, it suffices to show that

$$
2^{k-3} \lambda^{1-x_{0}} \geq \prod_{j=1}^{d-1}\left(1+\frac{1}{\lambda^{j-1}}\right)^{x_{j}}\left(k-2 x_{0}-2 \sum_{j=1}^{d-1} \frac{x_{j}}{1+\lambda^{j-1}}\right) .
$$

Recall that, according to the definition, each value $x_{j}$ is a non-negative integer. Note that the right side of inequality (6) and that of inequality (7) are both at most 0 if $x_{1} \geq k-2 x_{0}$; so we may assume that $x_{1} \in\left[k-2 x_{0}-1\right]$. Let us now fix $\lambda=4$, and prove the stronger statement that the right side of inequality $(7)$, which we call $R 8$, is always at most $2^{k-2 x_{0}-1}$. This implies both (6) and (7). Since the inequality is verified if $R 8 \leq 0$, we assume now on that $R 8$ is positive. We define $y_{j}:=\frac{x_{j}}{1+\lambda^{j-1}}$, for every $j \in\{1, \ldots, d-1\}$.

- If $x_{1}=k-2 x_{0}-1$, then

$$
\begin{aligned}
R 8 & =2^{k-2 x_{0}-1} \cdot \prod_{j=2}^{d-1}\left(1+\frac{1}{\lambda^{j-1}}\right)^{x_{j}}\left(1-2 \sum_{j=2}^{d-1} \frac{x_{j}}{1+\lambda^{j-1}}\right) & & \\
& =2^{k-2 x_{0}-1} \cdot \prod_{j=2}^{d-1}\left(1+\frac{1}{\lambda^{j-1}}\right)^{\left(1+\lambda^{j-1}\right) y_{j}}\left(1-2 \sum_{j=2}^{d-1} y_{j}\right) & & \\
& \leq 2^{k-2 x_{0}-1} \cdot \prod_{j=2}^{d-1}\left(\frac{3125}{1024}\right)^{y_{j}}\left(1-2 \sum_{j=2}^{d-1} y_{j}\right) & & \text { by Fact } 1 \text { because } R 8>0 \\
& =2^{k-2 x_{0}-1} \cdot\left(\frac{3125}{1024}\right)^{y}(1-2 y) & & \text { where } y:=\sum_{j=2}^{d-1} y_{j} \\
& \leq 2^{k-2 x_{0}-1} \cdot \max _{y \in \mathbb{R}^{+}}\left(\frac{3125}{1024}\right)^{y}(1-2 y) & & \text { because } R 8>0 \\
& \leq 2^{k-2 x_{0}-1} & & \text { by Fact } 2 .
\end{aligned}
$$

- If $x_{1}=k-2 x_{0}-2$, then

$$
R 8=2^{k-2 x_{0}-2} \cdot \prod_{j=2}^{d-1}\left(1+\frac{1}{\lambda^{j-1}}\right)^{x_{j}}\left(2-2 \sum_{j=2}^{d-1} \frac{x_{j}}{1+\lambda^{j-1}}\right) .
$$


If $x_{j}=0$ for every $j \in\{2, \ldots, d-1\}$, then $R 8 \leq 2^{k-2 x_{0}-1}$. Let us now assume otherwise, and set $j_{0}:=\min \left\{j \geq 2: x_{j}>0\right\}$. In particular $x_{j_{0}} \geq 1$ and $y_{j_{0}} \geq \frac{1}{1+\lambda^{j_{0}}-1}$. Then

$$
\begin{aligned}
& R 8=2^{k-2 x_{0}-2} \cdot \prod_{j=j_{0}}^{d-1}\left(1+\frac{1}{\lambda^{j-1}}\right)^{x_{j}}\left(2-2 \sum_{j=j_{0}}^{d-1} \frac{x_{j}}{1+\lambda^{j-1}}\right) \\
& =2^{k-2 x_{0}-2} \cdot \prod_{j=j_{0}}^{d-1}\left(1+\frac{1}{\lambda^{j-1}}\right)^{\left(1+\lambda^{j-1}\right) y_{j}}\left(2-2 \sum_{j=j_{0}}^{d-1} y_{j}\right) \\
& \leq 2^{k-2 x_{0}-2} \cdot \prod_{j=j_{0}}^{d-1}\left(1+\frac{1}{\lambda^{j_{0}-1}}\right)^{\left(1+\lambda^{j_{0}-1}\right) y_{j}}\left(2-2 \sum_{j=j_{0}}^{d-1} y_{j}\right) \quad \text { by Fact } 1 \text { because } R 8>0 \\
& =2^{k-2 x_{0}-2} \cdot\left(1+\frac{1}{\lambda^{j_{0}-1}}\right)^{\left(1+\lambda^{j_{0}-1}\right) y}(2-2 y) \quad \text { where } y:=\sum_{j=j_{0}}^{d-1} y_{j} \geq \frac{1}{1+\lambda^{j_{0}-1}} \\
& \leq 2^{k-2 x_{0}-2} \cdot \max _{y \geq \frac{1}{1+\lambda^{j} 0-1}}\left(1+\frac{1}{\lambda^{j_{0}-1}}\right)^{\left(1+\lambda^{j_{0}-1}\right) y}(2-2 y) \quad \text { because } R 8>0 \\
& \leq 2^{k-2 x_{0}-1} \\
& \text { by Fact } 2 \text {. }
\end{aligned}
$$

- If $x_{1} \leq k-2 x_{0}-3$, then

$$
\begin{array}{rlrl}
R 8 & =2^{x_{1}} \cdot \prod_{j=2}^{d-1}\left(1+\frac{1}{\lambda^{j-1}}\right)^{x_{j}}\left(k-2 x_{0}-x_{1}-2 \sum_{j=2}^{d-1} \frac{x_{j}}{1+\lambda^{j-1}}\right) & \\
& =2^{x_{1}} \cdot \prod_{j=2}^{d-1}\left(1+\frac{1}{\lambda^{j-1}}\right)^{\left(1+\lambda^{j-1}\right) y_{j}}\left(k-2 x_{0}-x_{1}-2 \sum_{j=2}^{d-1} y_{j}\right) & \\
& \leq 2^{x_{1}} \cdot \prod_{j=2}^{d-1}\left(\frac{3125}{1024}\right)^{y_{j}}\left(k-2 x_{0}-x_{1}-2 \sum_{j=2}^{d-1} y_{j}\right) & & \text { by Fact } 1 \text { because } R 8> \\
& =2^{x_{1}} \cdot\left(\frac{3125}{1024}\right)^{y}\left(k-2 x_{0}-x_{1}-2 y\right) & & \text { where } y:=\sum_{j=2}^{d-1} y_{j} \\
& \leq 2^{x_{1}} \cdot \max _{y \in \mathbb{R}}\left(\frac{3125}{1024}\right)^{y}\left(k-2 x_{0}-x_{1}-2 y\right) & & \text { because } R 8>0 \\
& \leq 2^{x_{1}} \frac{2\left(\frac{3125}{1024}\right)^{\frac{k-2 x_{0}-x_{1}}{2}}}{e \ln \left(\frac{3125}{1024}\right)} & & \text { by Fact } 2 \\
& \leq 2^{k-2 x_{0}-1} & & \text { as }\left(k-2 x_{0}-1\right) / 2>1 .
\end{array}
$$

This finishes to establish that

$$
\frac{2^{k-3}+k}{k} \mathbb{P}\left[v \in \mathbf{I}_{H} \mid E_{J}\right]+\frac{2}{k} \mathbb{E}\left[\left|N(v) \cap \mathbf{I}_{H}\right| \mid E_{J}\right] \geq 1 .
$$

The conclusion follows by the convex combination of (8) over all possible values of $J$.

We set $\lambda:=4$, and apply Lemma 1 with

$$
\alpha_{v}=1+\frac{2^{k(v)-3}}{k(v)} \quad \text { and } \quad \beta_{v}=\frac{2}{k(v)}
$$


for every vertex $v \in V(G)$, where $k(v)$ is chosen such that $\frac{2 \operatorname{deg}(v)+2^{k-3}}{k}$ is minimised when $k=k(v)$, and is always at least 4 since $\operatorname{deg}(v)$ is a non-negative integer. This ends the proof of Theorem 10

\section{Bounds on the Hall Ratio}

We focus on establishing upper bounds on the Hall ratios of graphs with bounded maximum degree and girth. These bounds are obtained by using the uniform distribution on $\mathscr{I}_{\alpha}(G)$, for $G$ in the considered class of graphs, into Lemma 4 or Lemma 5.

4.1. Structural analysis of a neighbourhood. We start by introducing some terminology.

\section{Definition 1.}

(1) A pattern of depth $r$ is any graph $P$ given with a root vertex $v$ such that

$$
\forall u \in V(G), \quad \operatorname{dist}_{G}(u, v) \leq r .
$$

The layer at depth $i$ of $P$ is the set of vertices at distance $i$ from its root vertex $v$.

(2) A pattern $P$ of depth $r$ and root $v$ is $d$-regular if all its vertices have degree exactly $d$, except maybe in the two deepest layers where the vertices have degree at most $d$.

Definition 2. Let $P$ be a pattern with depth $r$ and root $v$. Let $\mathbf{I}$ be a uniform random maximum independent set of $P$. We define $e_{i}(P):=\mathbb{E}\left[\left|\mathbf{I} \cap N_{P}^{i}(v)\right|\right]$ for each $i \in\{0, \ldots, r\}$.

(1) The constraint associated to $P$ is the pair $c(P)=(\mathbf{e}(P), n(P))$, where $\mathbf{e}(P)=\left(e_{i}(P)\right)_{i=0}^{r} \in$ $\left(\mathbb{Q}^{+}\right)^{r+1}$, and $n(P) \in \mathbb{N}$ is the cardinality of the constraint, which is the number of maximum independent sets of $P$. Most of the time, we only need to know the value of $\mathbf{e}(P)$, in which case we characterise the constraint $c(P)=(\mathbf{e}(P), n(P))$ only by $\mathbf{e}(P)$. The value of $n(P)$ is only needed for a technical reason, in order to be able to compute constraints inductively.

(2) Given two constraints $\mathbf{e}, \mathbf{e}^{\prime} \in\left(\mathbb{Q}^{+}\right)^{r+1}$, we say that $\mathbf{e}$ is weaker than $\mathbf{e}^{\prime}$ if, for any vector $\boldsymbol{\alpha} \in$ $\left(\mathbb{Q}^{+}\right)^{r+1}$ it holds that

$$
\boldsymbol{\alpha}^{\top} \mathbf{e}^{\prime} \geq 1 \quad \Longrightarrow \quad \boldsymbol{\alpha}^{\top} \mathbf{e} \geq 1
$$

If the above condition holds only for all vectors $\boldsymbol{\alpha} \in\left(\mathbb{Q}^{+}\right)^{r+1}$ with non-increasing coordinates, then we say that $\mathbf{e}$ is relatively weaker than $\mathbf{e}^{\prime}$.

Note that $\mathbf{e}$ is weaker than $\mathbf{e}^{\prime}$ if and only if

$$
\forall i \in\{0, \ldots, r\}, \quad e_{i} \geq e_{i}^{\prime},
$$

and $\mathbf{e}$ is relatively weaker than $\mathbf{e}^{\prime}$ if and only if

$$
\forall i \in\{0, \ldots, r\}, \quad \sum_{j=0}^{i} e_{j} \geq \sum_{j=0}^{i} e_{j}^{\prime} .
$$

Remark 2. Let $P$ be a pattern such that one of its vertices $u$ is adjacent with some vertices $u_{1}, \ldots, u_{k}$ of degree 1 in the next layer, where $k \geq 2$. Then every maximum independent set of $P$ contains $\left\{u_{1}, \ldots u_{k}\right\}$ and not $u$. Consequently, $\mathbf{e}(P)$ is weaker than $\mathbf{e}\left(P \backslash\left\{u_{3}, \ldots, u_{k}\right\}\right)$ since, letting $i$ be the distance between $u_{1}$ and the root of $P$, one has

$$
e_{j}(P)= \begin{cases}e_{j}\left(P \backslash\left\{u_{3}, \ldots, u_{k}\right\}\right) & \text { if } j \neq i, \text { and } \\ e_{i}\left(P \backslash\left\{u_{3}, \ldots, u_{k}\right\}\right)+(k-2) & \text { if } \mathrm{j}=\mathrm{i} .\end{cases}
$$

\subsection{Tree-like patterns.}


4.2.1. Rooting at a vertex. Fix an integer $r \geq 2$. Let $G$ be a $d$-regular graph of girth at least $2 r+2$, and let $\mathbf{I}$ be a uniform random maximum independent set of G. For any fixed vertex $v$, we let $J$ be any possible realisation of $\mathbf{I} \backslash N^{r}[v]$, and $W:=N^{r}[v] \backslash N(J)$. By the Spatial Markov Property of the uniform distribution over the maximum independent sets of $G$, the random independent set $\mathbf{I} \cap N^{r}[v]$ follows the uniform distribution over the maximum independent sets of $G[W]$. Now, observe that $G[W]$ is a $d$-regular pattern of depth $r$ with root vertex $v$, and since $G$ has girth at least $2 r+2$, this is a tree. Let $\mathcal{T}_{r}(d)$ be the set of acyclic $d$-regular patterns of depth $r$.

Let us define $\mathbf{X}_{i}(v):=\mathbf{I} \cap N^{i}(v)$, for every $i \in\{0, \ldots, r\}$. We seek parameters $\left(\alpha_{i}\right)_{i=0}^{r}$ such that the inequality $\sum_{i=0}^{r} \alpha_{i} \mathbb{E}\left[\left|\mathbf{X}_{i}(v)\right|\right] \geq 1$ is satisfied regardless of the choice of $v$. To this end, it is enough to pick the rational numbers $\alpha_{i}$ in such a way that the inequality is satisfied in any tree $T \in \mathcal{T}_{r}(d)$, when $v$ is the root vertex. In a more formal way, given any $T \in \mathcal{T}_{r}(d)$, the vector $\boldsymbol{\alpha}=\left(\alpha_{0}, \ldots, \alpha_{r}\right)$ must be compatible with the constraint $\mathbf{e}(T)$, that is, $\boldsymbol{\alpha}^{\top} \mathbf{e}(T) \geq 1$ for each $T \in \mathcal{T}_{r}(d)$.

An application of Lemma 4 then lets us conclude that the desired bound is the solution to the following linear program.

$$
\begin{gathered}
\qquad \frac{|G|}{\alpha(G)} \leq \min \quad \alpha_{0}+\sum_{i=1}^{r} \alpha_{i} d(d-1)^{i-1} \\
\text { such that } \begin{cases}\forall T \in \mathcal{T}_{r}(d), & \sum_{i=0}^{r} \alpha_{i} e_{i}(T) \geq 1 \\
\forall i \leq r, & \alpha_{i} \geq 0 .\end{cases}
\end{gathered}
$$

The end of the proof is made by computer generation of $\mathcal{T}_{r}(d)$, in order to generate the desired linear program, which is then solved again by computer computation. For the sake of illustration, we give a complete human proof of the case where $r=2$ and $d=3$. There are 10 trees in $\mathcal{T}_{2}(3)$. One can easily compute the constraint $\left(e_{0}(T), e_{1}(T), e_{2}(T)\right)$ for each $T \in \mathcal{T}_{2}(3)$; they are depicted in Figure 4. Note that constraints $\mathbf{e}_{8}, \mathbf{e}_{9}$ and $\mathbf{e}_{10}$ are weaker than constraint $\mathbf{e}_{7}$, so we may disregard these constraints in the linear program to solve. Note also that constraint $\mathbf{e}_{0}$ is relatively weaker than constraint $\mathbf{e}_{1}$, and so may be disregarded as well, provided that the solution of the linear program is attained by a vector $\boldsymbol{\alpha}$ with non-increasing coordinates, which will have to be checked. The linear program to solve is therefore the following.

$$
\begin{array}{cl}
\operatorname{minimise} & \begin{array}{l}
\alpha_{0}+3 \alpha_{1}+6 \alpha_{2} \\
\text { such that }
\end{array} \\
\begin{array}{c}
5 / 2 \cdot \alpha_{1}+1 / 2 \cdot \alpha_{2} \\
2 \alpha_{1}+2 \alpha_{2}
\end{array} & \geq 1 \\
1 / 5 \cdot \alpha_{0}+8 / 5 \cdot \alpha_{1}+6 / 5 \cdot \alpha_{2} & \geq 1 \\
1 / 3 \cdot \alpha_{0}+\alpha_{1}+8 / 3 \cdot \alpha_{2} & \geq 1 \\
1 / 2 \cdot \alpha_{0}+1 / 2 \cdot \alpha_{1}+4 \alpha_{2} & \geq 1 \\
\alpha_{0}+3 \alpha_{2} & \geq 1 \\
\alpha_{0}, \alpha_{1}, \alpha_{2} \geq 0 . &
\end{array}
$$

The solution of this linear program is $\frac{85}{31} \approx 2.741935$, attained by $\boldsymbol{\alpha}=\left(\frac{19}{31}, \frac{14}{31}, \frac{4}{31}\right)$, which indeed has non-increasing coordinates. This is an upper bound on $\rho(3,6)$, though we prove a stronger one through a more involved computation in Section 4.3.2.

To compute $\mathbf{e}(T)$ for each $T \in \mathcal{T}_{r}(d)$, one can enumerate all the maximum independent sets of $T$ and average the size of their intersection with each layer of $T$. For general graphs, there might 


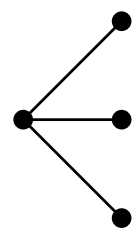

$\mathbf{e}_{1}=(0,3,0)$

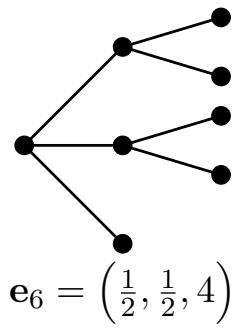

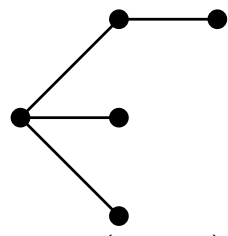

$\mathbf{e}_{2}=\left(0, \frac{5}{2}, \frac{1}{2}\right)$

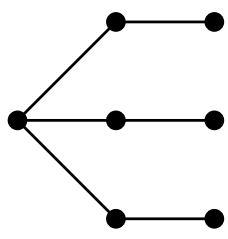

$\mathbf{e}_{7}=(1,0,3)$

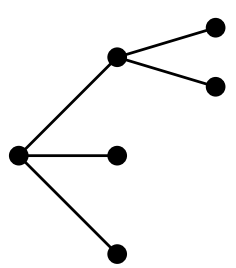

$\mathbf{e}_{3}=(0,2,2)$

$\mathbf{e}_{4}=\left(\frac{1}{5}, \frac{8}{5}, \frac{6}{5}\right)$
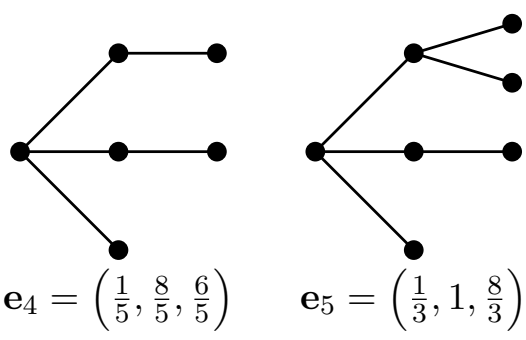

$\mathbf{e}_{5}=\left(\frac{1}{3}, 1, \frac{8}{3}\right)$
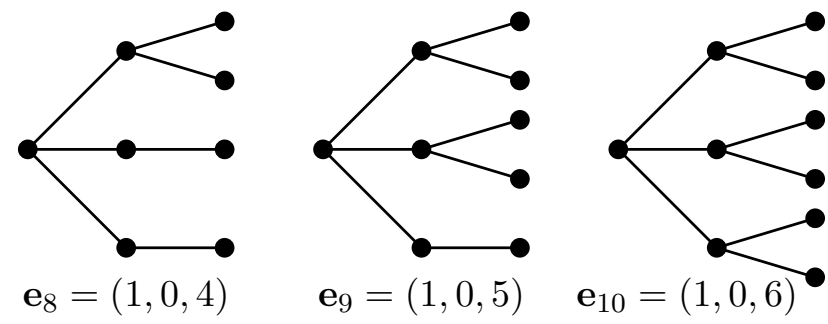

Figure 4. An enumeration of $\mathbf{e}(T)$ for all trees $T \in \mathcal{T}_{2}(3)$.

be no better way of doing so, however the case of $\mathcal{T}_{r}(d)$ can be treated inductively by a standard approach: we distinguish between the maximum independent sets that contain the root and those that do not. We introduce the following notation; to this end, we slightly extend our notion of "constraint" to any pair $(\mathbf{e}, n)$ where $\mathbf{e} \in\left(\mathbb{Q}^{+}\right)^{r+1}$ and $n$ is a non-negative integer; the constraints we shall use have a combinatorial interpretation with respect to some pattern.

Definition 3. Let $c=(\mathbf{e}, n)$ and $c^{\prime}=\left(\mathbf{e}^{\prime}, n^{\prime}\right)$ be two constraints.

(1) The operation $\vee$ on $c$ and $c^{\prime}$ returns the constraint

$$
c \vee c^{\prime}:= \begin{cases}\left(\frac{n}{n+n^{\prime}} \mathbf{e}+\frac{n^{\prime}}{n+n^{\prime}} \mathbf{e}^{\prime}, n+n^{\prime}\right) & \text { if }\|\mathbf{e}\|_{1}=\left\|\mathbf{e}^{\prime}\right\|_{1}, \\ c & \text { if }\|\mathbf{e}\|_{1}>\left\|\mathbf{e}^{\prime}\right\|_{1}, \\ c^{\prime} & \text { if }\|\mathbf{e}\|_{1}<\left\|\mathbf{e}^{\prime}\right\|_{1} .\end{cases}
$$

(2) The operation $\oplus$ on $c$ and $c^{\prime}$ returns the constraint $c \oplus c^{\prime}:=\left(\mathbf{e}+\mathbf{e}^{\prime}, n \cdot n^{\prime}\right)$.

For a given tree $T \in \mathcal{T}_{r}(d)$ with root $v$, let $c_{1}(T)$ be the constraint associated to $T$ where $v$ is forced (that is, we restrict to the maximum independent sets that contain $v$ when computing the constraint $c_{1}(T)$ ), and let $c_{0}(T)$ be the constraint associated to $T$ where $v$ is forbidden. It readily follows from Definition 3 that

$$
c(T)=c_{0}(T) \vee c_{1}(T) .
$$

If $\left(T_{i}\right)_{i=1}^{d}$ are the subtrees of $T$ rooted at the children of the root $v$ (some of which might be empty), then

$$
\begin{array}{ll}
c_{0}(T)=((0, \mathbf{e}), n) & \text { where }(\mathbf{e}, n)=\bigoplus_{i=1}^{d} c\left(T_{i}\right), \quad \text { and } \\
c_{1}(T)=((1, \mathbf{e}), n) & \text { where }(\mathbf{e}, n)=\bigoplus_{i=1}^{d} c_{0}\left(T_{i}\right) .
\end{array}
$$

We thus obtain an inductive way of computing $\mathbf{e}(T)$ by using the following initial values. 


$$
\begin{aligned}
c_{0}(\varnothing) & :=((0), 1) \\
c_{0}(\{v\}) & :=((0), 1)
\end{aligned}
$$

$$
\begin{aligned}
c_{1}(\varnothing) & :=((0), 0) \\
c_{1}(\{v\}) & :=((1), 1) .
\end{aligned}
$$

Using this inductive way to enumerate the vectors $\mathbf{e}(T)$ for $T \in \mathcal{T}_{r}(d)$, the following statement is obtained by computer calculus.

Lemma 8. The solution to the linear program (9) is

$$
\begin{aligned}
& \mathcal{T}_{3}(3): \quad \frac{5849}{2228} \approx 2.625224 \quad \text { with } \boldsymbol{\alpha}=\left(\frac{953}{2228}, \frac{162}{557}, \frac{81}{557}, \frac{21}{557}\right), \\
& \mathcal{T}_{4}(3): \quad \frac{2098873192}{820777797} \approx 2.557176 \quad \text { with } \boldsymbol{\alpha}=\left(\frac{225822361}{820777797}, \frac{18575757}{91197533}, \frac{10597368}{91197533},\right. \\
& \left.\frac{5054976}{91197533}, \frac{1172732}{91197533}\right) \\
& \mathcal{T}_{5}(3): \quad \frac{29727802051155412}{11841961450578397} \approx 2.510378 \text { with } \boldsymbol{\alpha}=\left(\frac{3027359065168972}{11841961450578397}, \frac{2216425114872980}{11841961450578397},\right. \\
& \left.\frac{2224040336719575}{23683922901156794}, \frac{2026654050681425}{47367845802313588}, \frac{403660478424775}{23683922901156794}, \frac{51149140376400}{11841961450578397}\right), \\
& \mathcal{T}_{3}(4): \quad \frac{7083927}{2331392} \approx 3.038497 \quad \text { with } \boldsymbol{\alpha}=\left(\frac{123345}{333056}, \frac{68295}{291424}, \frac{12283}{145712}, \frac{2911}{145712}\right), \\
& \mathcal{T}_{4}(4): \quad 3 \\
& \mathcal{T}_{2}(5): \quad \frac{69}{19} \approx 3.631579 \quad \text { with } \boldsymbol{\alpha}=\left(\frac{37}{57}, \frac{6}{19}, \frac{4}{57}\right), \\
& \mathcal{T}_{3}(5): \quad \frac{7}{2}=3.5 \quad \text { with } \boldsymbol{\alpha}=\left(\frac{77}{282}, \frac{25}{141}, \frac{17}{282}, \frac{2}{141}\right) .
\end{aligned}
$$

4.2.2. Rooting in an edge. Definition 1 can be extended to a pattern with a root-edge instead of a root-vertex. The distance in a pattern $P$ between a vertex $w$ and an edge $u v$ is defined to be $\min \left\{\operatorname{dist}_{P}(w, u), \operatorname{dist}_{P}(w, v)\right\}$. The depth of a pattern $P$ rooted in an edge $e$ is then the largest distance between $e$ and a vertex in $P$. It is possible to follow the same analysis as in Section 4.2.1 with edge-rooted patterns: in order for the edge-rooted pattern of depth $r$ to always be a tree, the graph $G$ must have girth at least $2 r+3$. Let $\mathcal{T}_{r}^{\prime}(d)$ be the set of acyclic edge-rooted $d$-regular patterns of depth $r$. By Lemma 5, the linear program to solve is now the following.

$$
\begin{gathered}
\frac{|G|}{\alpha(G)} \leq \min \quad 2 \sum_{i=0}^{r} \alpha_{i}(d-1)^{i} \\
\text { such that } \begin{cases}\forall T \in \mathcal{T}_{r}^{\prime}(d), & \sum_{i=0}^{r} \alpha_{i} e_{i}(T) \geq 1 \\
\forall i \leq r, & \alpha_{i} \geq 0 .\end{cases}
\end{gathered}
$$

For a given tree $T \in \mathcal{T}_{r}^{\prime}(d)$ rooted in $e=u v$, it is possible to compute $\mathbf{e}(T)$ using the constraints associated to vertex-rooted trees. If $T_{u}$ and $T_{v}$ are the subtrees of $T$ respectively rooted at $u$ and at $v$, then it readily follows from Definition 3 that

$$
c(T)=\left(c_{0}\left(T_{u}\right) \oplus c_{0}\left(T_{v}\right)\right) \vee\left(c_{0}\left(T_{u}\right) \oplus c_{1}\left(T_{v}\right)\right) \vee\left(c_{1}\left(T_{u}\right) \oplus c_{0}\left(T_{v}\right)\right) .
$$


Following the enumeration of the vectors $\mathbf{e}(T)$ for $T \in \mathcal{T}_{r}^{\prime}(d)$ described earlier, the next statement is obtained by computer calculus.

Lemma 9. The solution to the linear program $(10)$ is

$$
\begin{array}{lll}
\mathcal{T}_{2}^{\prime}(3): & \frac{30}{11} \approx 2.72727 & \text { with } \boldsymbol{\alpha}=\left(\frac{1}{2}, \frac{13}{44}, \frac{3}{44}\right), \\
\mathcal{T}_{3}^{\prime}(3): & \frac{125}{48} \approx 2.604167 & \text { with } \boldsymbol{\alpha}=\left(\frac{11}{32}, \frac{5}{24}, \frac{3}{32}, \frac{1}{48}\right), \\
\mathcal{T}_{4}^{\prime}(3): & \frac{14147193}{5571665} \approx 2.539132 & \text { with } \boldsymbol{\alpha}=\left(\frac{98057}{506515}, \frac{159348}{1114333}, \frac{3688469}{44573320}, \frac{1752117}{44573320}, \frac{402569}{44573320}\right), \\
\mathcal{T}_{2}^{\prime}(4): & \frac{41}{13} \approx 3.153846 & \text { with } \boldsymbol{\alpha}=\left(\frac{11}{26}, \frac{3}{13}, \frac{2}{39}\right), \\
\mathcal{T}_{3}^{\prime}(4): & \frac{127937}{42400} \approx 3.017382 & \text { with } \boldsymbol{\alpha}=\left(\frac{5539}{16960}, \frac{1737}{10600}, \frac{257}{5300}, \frac{399}{42400}\right), \\
\mathcal{T}_{2}^{\prime}(5): & \frac{18}{5}=3.6 & \text { with } \boldsymbol{\alpha}=\left(\frac{17}{45}, \frac{8}{45}, \frac{2}{45}\right) .
\end{array}
$$

The bounds obtained in Lemma 9 are valid for graphs of girth at least $2 r+3$. It turns out that the same bounds, with the same $\boldsymbol{\alpha}$, remain valid for graphs of girth $2 r+2=6$, when $r=2$ and $d \in\{3,4\}$. We were not able to check this for higher values of $r$ or $d$, but we propose the following conjecture which would explain and generalise this phenomenon.

Conjecture 2. Let $P$ be a d-regular edge-rooted pattern of depth $r$ and of girth $2 r+2$. Then the constraint $\mathbf{e}(P)$ is weaker than some convex combination of constraints $\mathbf{e}(T)$ with $T \in \mathcal{T}_{r}^{\prime}(d)$. More formally, there exist $T_{1}, \ldots, T_{m} \in \mathcal{T}_{r}^{\prime}(d)$ and $\lambda_{1}, \ldots, \lambda_{m} \in[0,1]$ with $\sum_{i=1}^{m} \lambda_{i}=1$ such that for any $\boldsymbol{\alpha} \in\left(\mathbb{Q}^{+}\right)^{r+1}$,

$$
\boldsymbol{\alpha}^{\top}\left(\sum_{i=1}^{m} \lambda_{i} \mathbf{e}\left(T_{i}\right)\right) \geq 1 \quad \Longrightarrow \quad \boldsymbol{\alpha}^{\top} \mathbf{e}(P) \geq 1 .
$$

\subsection{More complicated patterns.}

4.3.1. Rooting at a vertex. Let us fix a depth $r \geq 2$. Let $G$ be a $d$-regular graph of girth $g \leq 2 r+1$. We repeat the same analysis as in Section 4.2.1. we end up having to find a vector $\boldsymbol{\alpha} \in \mathbb{Q}^{r+1}$ compatible with all the constraints generated by vertex-rooted $d$-regular patterns of depth $r$ and girth $g$. Letting $\mathcal{P}_{r}(d, g)$ be the set of such patterns, we thus want that

$$
\forall P \in \mathcal{P}_{r}(d, g), \quad \boldsymbol{\alpha}^{\top} \mathbf{e}(P) \geq 1 .
$$

In this setting, we could do no better than performing an exhaustive enumeration of every possible pattern $P \in \mathcal{P}_{r}(d, g)$, and computing the associated constraint $\mathbf{e}(P)$ through an exhaustive enumeration of $\mathscr{I}_{\alpha}(P)$. The complexity of such a process grows fast, and we considered only depth $r \leq 2$ and degree $d \leq 4$. Since the largest value of the Hall ratio over the class of 3-regular graphs of girth 4 or 5 is known to be $\frac{14}{5}=2.8$, and the one of 4-regular graphs of girth 4 is known to be $\frac{13}{4}=3.25$, the only open value in these settings is for the class of 4-regular graphs of girth 5 . Unfortunately, this method is not powerful enough to prove an upper bound lower than $\frac{13}{4}$, the obtained bound for $\mathcal{P}_{2}(4,5)$ being $\frac{82}{25}=3.28$. It is more interesting to root the patterns in an edge.

4.3.2. Rooting in an edge. Similarly, we define $\mathcal{P}_{r}^{\prime}(d, g)$ to be the set of edge-rooted $d$-regular patterns of girth $g$. For fixed $r$ and $g$, we seek for the solution of the following linear program. 


$$
\begin{gathered}
\frac{|G|}{\alpha(G)} \leq \min \quad 2 \sum_{i=0}^{r} \alpha_{i}(d-1)^{i} \\
\text { such that } \begin{cases}\forall P \in \mathcal{P}_{r}^{\prime}(d, g), & \sum_{i=0}^{r} \alpha_{i} e_{i}(P) \geq 1 \\
\forall i \leq r, & \alpha_{i} \geq 0 .\end{cases}
\end{gathered}
$$

Again, our computations were limited to the cases where $r \leq 2$ and $d \leq 4$. However, we managed to prove improved bounds for girth 6 when $d \in\{3,4\}$, which seems to support Conjecture 2

Lemma 10. The solution to the linear program (12) is

$$
\begin{array}{lll}
\mathcal{P}_{2}^{\prime}(3,6): & \frac{30}{11} \approx 2.72727 & \text { with } \boldsymbol{\alpha}=\left(\frac{1}{2}, \frac{13}{44}, \frac{3}{44}\right), \\
\mathcal{P}_{2}^{\prime}(4,6): & \frac{41}{13} \approx 3.153846 & \text { with } \boldsymbol{\alpha}=\left(\frac{11}{26}, \frac{3}{13}, \frac{2}{39}\right) .
\end{array}
$$

\section{Conclusion}

We finish with a discussion about our method, its possible future applications, and its limitations.

5.1. Going further. We have decided to restrict ourselves in this work to graphs with given girth. Our general framework reduces the remaining work to an analysis of the behaviour of the hardcore distribution in trees of a given depth, which minimises the need to use massive computations through a computer in order to derive our results. In the future, it will be interesting to apply our framework to other classes of graphs. We can imagine any class of graphs which is locally constrained, that is graphs of maximum degree $d$ whose neighbourhoods up to a fixed depth $r$ is a strict subset of graphs of maximum degree $d$ and radius $r$. For instance, one could consider the Hall ratio and fractional chromatic number of $C_{\ell}$-free graphs for a fixed value of $\ell$, of $K_{4}$-free graphs, or of squares of graphs of girth at least some constant $g$.

It would be interesting to be able to feed our framework with other probability distributions on the independent sets. A promising one would be the following: let $\mathbf{I}$ be a random maximal independent set obtained greedily by fixing a uniform random priority ordering on the vertices. Shearer [21] observed that $\mathbb{E}[\mathbf{I}]$ matches the bound given in Theorem 2 . However, such a distribution has a strong global dependency; it might be really hard - if possible at all — but probably fruitful, to find a spatial Markov property satisfied by this distribution.

5.2. On the hard-core model. In the present work, we have fed our method with the hard-core distribution at different regimes. We have considered all the independent sets with a fugacity $\lambda \rightarrow 0$, restricted ourselves to the maximal independent sets with a fugacity $\lambda=4$, and then to the maximum independent sets, which forces the uniform distribution with $\lambda=\infty$.

We observe that when we let the fugacity $\lambda$ grow, and hence favour larger independent sets, we obtain better bounds for really small values of the degree. On the other hand, we are more constrained in our choices, which translates into worse asymptotic bounds.

It could be unsettling that restricting to maximal independent sets does not yield a strict improvement in the bound obtained with our method; indeed it is always possible to find an optimal fractional colouring using only maximal independent sets as colour classes, up to covering some vertices with weight more that 1 . However, because we fix the realisation of the random independent set on a large part of the graph, we suffer from a deeper propagation of the constraints due to the maximality of the independent set. When working with a partial realisation of a maximal independent set, vertices at distance 2 from that realisation can be forbidden, hence the need to 
work with deeper patterns (we must always have the freedom to pick the root of the pattern in the independent set).

Another unsettling observation comes from the fact that the optimised fugacity when working with the whole set of independent sets is $\lambda \rightarrow 0$ as the degree $d \rightarrow \infty$. This means that the independent set which receives the largest weight in our fractional colouring is the empty independent set, which seems rather wasteful. This is however needed in order to assign a non negligible weight to the independent set that contains only the root (when we work with patterns of depth 1). Indeed, there is only one independent set that contains the root, while there are exponentially many independent sets that do not contain it. This is no longer the case when we restrict to maximal independent sets, which explains why the optimal value of the fugacity is a constant in that case.

5.3. Limitations of the method. While our framework gives a lot of freedom in its possible applications, it still suffers from some limitations which we discuss hereafter.

We cannot improve the bounds for the fractional chromatic number by trying to increase the girth. Indeed, since the class of patterns that we need to consider in our framework must be hereditary in that case, increasing the depth would only strictly increase the number of patterns (the ones of smaller depth are still present). Therefore, the girth of the class of graphs for which we can derive our bound is determined by the choice of the probability distribution, and the depth of its dependencies.

Working with maximum independent sets, it appears that our method cannot prove a better bound for $\rho(d, \infty)$ than $d / 2+1$, which is reached at some girth $g_{0}$. We could not find an explanation for this boundary, although such an explanation would have a strong theoretical interest. So, in order to obtain new bounds with our method for $\rho(d, g)$, one would have to use the hard-core model in different regimes than the one where $\lambda=\infty$.

The bounds obtained appear not to be tight, if we consider the pairs $(d, g)$ for which we know the value of $\rho$ and $\chi_{f}$ and compare it with the bound obtained using our method. However, obtaining these tight bounds systematically requires a deep structural analysis already when $d=3$, and an even deeper one when $d=4$. It is unlikely that these structural analyses could be generalised in order to cover larger values of $d$, while our method provides a smooth transition between the small values of $d$ and the asymptotic regime.

5.4. Note added. After this work first appeared in a public preprint repository, a work from Cames van Batenburg, Goedgebeur, Joret [6] improved our bound for $\rho(3,6)$, by establishing that it is at most $8 / 3$. This work relies again on a deep structural analysis of cubic graphs avoiding some finite family of graphs (all of which contain a $C_{5}$ ) as a subgraph.

5.5. Acknowledgement. We are thankful to Ewan Davies for the insightful discussion about the behaviour of the hard-core distribution on closed neighbourhoods, which led to Theorem 9.

\section{REFERENCES}

[1] B. Bajnok AND G. BRInkmann, On the independence number of triangle free graphs with maximum degree three, J. Combin. Math. Combin. Comput., 26 (1998), pp. 237-254.

[2] C. BERge, Färbung von graphen, deren sämtliche bzw. deren ungerade kreise starr sind (zusammenfassung), Wiss. Z. Martin-Luther Univ. Halle-Wittenberg, Math.-Natur. Reihe, 10 (1961), pp. 114-115.

[3] N. BigGs, Constructions for cubic graphs with large girth, Electron. J. Combin., 5 (1998). \#A1.

[4] B. Bollobás, The independence ratio of regular graphs, Proc. Amer. Math. Soc., 83 (1981), pp. $433-436$.

[5] R. L. Brooks, On colouring the nodes of a network, Proc. Cambridge Philos. Soc., 37 (1941), pp. $194-197$.

[6] W. Cames van Batenburg, G. Joret, J. Goedgebeur, Large independent sets in triangle-free cubic graphs: beyond planarity, Advances in Combinatorics, P7, 2020.

[7] M. Chudnovsky, N. Robertson, P. D. Seymour, and R. Thomas, The strong perfect graph theorem, Ann. of Math. (2), 164 (2006), pp. 51-229. 
[8] E. Davies, R. De Joannis de Verclos, R. J. Kang, And F. Pirot, Colouring triangle-free graphs with local list sizes, arXiv e-prints, (2018).

[9] Z. DvořÁk, J.-S. Sereni, J. Volec, Subcubic triangle-free graphs have fractional chromatic number at most 14/5, Journal of the London Mathematical Society, Volume 89, Issue 3, pp. 641-662, 2014.

[10] P. ERdős, Some remarks on the theory of graphs, Bull. Amer. Math. Soc., 53(4):292-294,04, 1947.

[11] P. Erdős And G. Szekeres, A Combinatorial Problem in Geometry, Compos. Math. 2(1935), pp. 463-470.

[12] G. Exoo And R. JAJCAY, Dynamic cage survey, Elec. J. Combin., Dynamic Surveys (2008). \#DS16.

[13] K. Fraughnaugh and S. C. Locke, 11/30 (finding large independent sets in connected triangle-free 3-regular graphs), J. Combin. Theory Ser. B, 65 (1995), pp. 51-72.

[14] K. F. Jones, Independence in graphs with maximum degree four, J. Combin. Theory Ser. B, 37 (1984), pp. 254269.

[15] F. Kardoš, D. Král', And J. Volec, Fractional colorings of cubic graphs with large girth, SIAM J. Discrete Math., 25 (2011), pp. 1454-1476.

[16] A. D. KING, Claw-free graphs and two conjectures on $\omega, \Delta$, and $\chi$, ph.d. thesis, McGill University, 2009.

[17] A. V. KostochkA, Degree, girth and chromatic number, in Combinatorics (Proc. Fifth Hungarian Colloq., Keszthely, 1976), Vol. II, vol. 18 of Colloq. Math. Soc. János Bolyai, North-Holland, Amsterdam-New York, 1978, pp. 679-696.

[18] M. Molloy, The list chromatic number of graphs with small clique number, J. Combin. Theory Ser. B, 134 (2019), pp. 264-284.

[19] M. Molloy And B. A. Reed, Graph colouring and the probabilistic method, vol. 23 of Algorithms and Combinatorics, Springer-Verlag, Berlin, 2002.

[20] F. P. RAmsey., On a problem of formal logic, Proceedings of the London Mathematical Society, s2-30(1):264-286, 1930.

[21] J. B. Shearer, A note on the independence number of triangle-free graphs, Discrete Math.,46(1):83-87, 1983.

[22] J. B. ShEARER, A note on the independence number of triangle-free graphs, ii, Journal of Combinatorial Theory, Series B, 53 (1991), pp. 300-307.

[23] W. Staton, Some Ramsey-type numbers and the independence ratio, Trans. Amer. Math. Soc., 256 (1979), pp. $353-370$.

Équipe Orpailleur, LORIA (Université de Lorraine, C.N.R.S., INRIA), Vandeuvre-LÈs-NanCy, France and Department of Mathematics, Radboud University Nijmegen, Netherlands.

Email address: francois.pirot@loria.fr

Centre National de la Recherche Scientifique (ICube, CSTB), Strasbourg, France.

Email address: sereni@kam.mff.cuni.cz 